\title{
The Status of the Minimum Principle in the Theoretical Analysis of Visual Perception
}

\author{
Gary Hatfield \\ Department of Philosophy \\ Johns Hopkins University
}

\author{
William Epstein \\ University of Wisconsin—Madison
}

\begin{abstract}
We examine a number of investigations of perceptual economy or, more specifically, of minimum tendencies and minimum principles in the visual perception of form, depth, and motion. A minimum tendency is a psychophysical finding that perception tends toward simplicity, as measured in accordance with a specified metric. A minimum principle is a theoretical construct imputed to the visual system to explain minimum tendencies. After examining a number of studies of perceptual economy, we embark on a systematic analysis of this notion. We examine the notion that simple perceptual representations must be defined within the "geometric constraints" provided by proximal stimulation. We then take up metrics of simplicity. Any study of perceptual economy must use a metric of simplicity; the choice of metric may be seen as a matter of convention, or it may have deep theoretical and empirical implications. We evaluate several answers to the question of why the visual system might favor economical representations. Finally, we examine several accounts of the process for achieving perceptual economy, concluding that those which favor massively parallel processing are the most plausible.
\end{abstract}

The notions of "simplicity" and "economy" have been used in varied contexts within the sciences (see Sober, 1975). It was a commonplace of classical physics and astronomy that "nature acts by the simplest means." Euler, Lagrange, Hamilton, and others have shown that the central equations of mechanics can be formulated isoperimetrically (in terms of maximum/minimum solutions). In a broader vein, methodologists have proposed that scientists proceed in accordance with the principle of parsimony, which holds that of two theories with equal empirical adequacy, the simpler theory should be chosen. A century ago Mach (1883/1960, 1919) referred this principle to a psychological preference of the scientific investigator for economy of thought. Finally, psychologists have found a tendency

Support for this research was provided by a fellowship awarded to Gary Hatfield by the American Council of Learned Societies and by a grant to William Epstein from the Wisconsin Alumni Research Foundation. The authors express their gratitude to W. Anderson, J. Cutting, L. J. Daston, H. Egeth, J. E. Hochberg, S. M. Kosslyn, T. J. Sejnowski, and E. Sober for their comments, criticism, and discussion of various drafts.

Requests for reprints should be sent to Gary Hatfield, Department of Philosophy, Johns Hopkins University, Baltimore, Maryland 21218. in perception toward phenomenal simplicity and regularity.

It is only natural that connections should be made among these diverse concerns with simplicity by virtue of their common label. However, as useful as it may be heuristically to see these diverse concerns as manifestations of a single principle of economy, one must keep in mind that what is meant by "simplicity" in a scientific context is closely related to how one measures it. A general result of philosophical treatments of simplicity is the discovery that simplicity metrics (e.g., for measuring the simplicity of theories or other descriptions of the world) are highly sensitive to oftentimes arbitrary terminological conventions within a theoretical or other descriptive vocabulary; no universally applicable simplicity metric is close to formulation (Goodman, 1972). Given the current means for measuring and comparing instances of "simplicity," it is possible that simplicity as manifested in, say, mechanics and perception are merely analogically related. Sweeping claims that see in all of the mentioned phenomena the operation of a single Minimum Principle must be treated with great caution.

Within the psychology of perception, diverse theoretical approaches have led to dif- 
fering conceptions of perceptual economy itself, which may be grouped into three categories. The first category is the notion that perceived objects will tend toward phenomenal simplicity: All else being equal, the object of the perceptual experience will have, for example, the simplest shape possible. This notion is connoted by the terminology of "good form" or "Prägnanz." The second category is descriptive economy: Here the idea is that the objects of perceptual experience will be such that they can be described using the fewest predicates in a given language (perhaps English, but more likely a symbol system developed especially for describing percepts). This conception of simplicity in perception is often characterized as "informational economy." Finally, there is the notion of economy of process: All else being equal, the object of perceptual experience will be the one that results from the most economical internal process. This emphasis is reflected in references to "minimum processing load," but also in the Gestalt notion of prägnant physiological processes. Our approach to the topic of perceptual economy will emphasize simplicity in the phenomenal organization of perceived objects and events, although any approach to this topic must take up economy of description and economy of process.

Investigations during the past three decades (Hochberg \& McAlister, 1953, to Buffart, Leeuwenberg, \& Restle, 1983) have shown that perceived objects exhibit a tendency toward minimum diversity and change or toward maximum regularity and simplicity (as measured by various metrics of simplicity). We label these diverse psychophysical findings that perception tends toward economy or simplicity instances of various minimum tendencies. A number of studies have been conducted to establish empirically whether perception in fact exhibits minimum tendencies, without seeking to establish any particular explanation of such tendencies (e.g., Hochberg \& Brooks, 1960; Hochberg \& McAlister, 1953).

In addition to studies motivated chiefly by empirical goals, a number of investigators have sought to develop an explanation of the observed minimum tendencies. Two types of explanatory problems arise: (a) Why does the visual system operate in such a way that perceptual economy is achieved? (b) How does the visual system achieve this economy? The first question concerns the origin of minimum tendencies, and it seeks an answer within a broad theoretical approach to the explanation of the behavior of the perceptual system. The second question concerns the actual processes that result in the manifestation of minimum tendencies, and it requires an answer in terms of specific processing mechanisms.

Regarding the origin of minimum tendencies, some investigators have suggested that these tendencies reveal the operation of a cardinal principle of perceptual processing. As illustration, Hochberg (1964) suggested that a minimum principle could provide the foundations of a general psychophysics of space, and thus yield general explanations of form and depth perception. This suggestion is reminiscent of the position of the Gestalt psychologists, who believed that a minimum principle could provide a unified explanation of a broad range of empirical findings, including figure-ground organization, shape perception, and depth perception (Koffka, 1935, chap. 4). Other investigators admit minimum tendencies at the empirical level but do not seek their explanation in a general minimum principle. Hochberg $(1974,1981)$ and Perkins (1976) attributed minimum tendencies to the operation of a likelihood or hypothesis-formation principle. Perkins argued that minimum solutions are favored because they are a "good bet" (a likely hypothesis) about the actual properties of objects in the environment.

The goals of this article are threefold. The first is to gather the various strands of recent empirical investigation into minimum tendencies for purposes of comparison and evaluation, with an eye toward discerning common approaches and common problems. Local criticisms are presented along the way. Our second goal is to explore the theoretical status of minimum tendencies and proposed minimum principles. Here we engage in systematic critical analysis. Concern with theoretical formulations leads to our third goal: consideration of the chief types of process models proposed to account for minimization. 


\section{Investigation of Minimum Tendencies and Minimum Principles in Contemporary Psychology}

Any evaluation of minimum tendencies and the minimum principles in contemporary psychology must begin with their empirical status. Minimum tendencies have been examined in a variety of empirical settings, including the perception of form, depth, and motion configurations. The theoretical questions suggested by various attempts to investigate minimum tendencies empirically are noted as they arise.

\section{Perception of Form and Depth}

Hochberg and McAlister. Hochberg and McAlister (1953) should be credited with initiating the efforts to place the notoriously qualitative notion of figural "goodness" on a quantitative footing (see also Attneave, 1954). These investigators used line drawings which could give rise to alternative perceptual organizations, and they assumed that the "better" of these organizations would be perceived more often or for a longer span of time than the alternatives. They predicted that "goodness," as defined by response frequency, would be correlated with informational economy as determined by their postulated metric of "information": "the less the amount of information needed to define a given [perceptual] organization as compared to other alternatives, the more likely that the figure will be so perceived" (Hochberg \& McAlister, 1953, p. 361). A test of this formulation requires an objective specification of the informational economy of rival percepts and the means for ascertaining the likelihood of these percepts.

Hochberg and McAlister's (1953) treatment is best understood by considering the four patterns they adopted for study (see Figure 1). Each drawing may be seen either as a bidimensional patterned hexagon or as a tridimensional cube. The authors proposed that the four patterns differ with respect to the amount of information needed to specify each as a bidimensional pattern. The amount of information was determined by counting line segments, angles, and points of intersec-
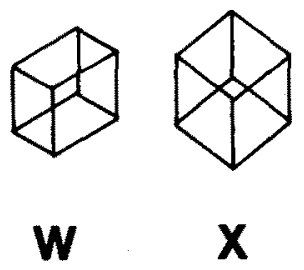
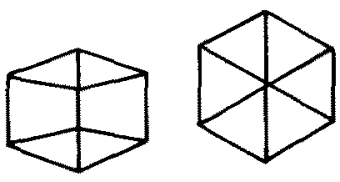

Figure 1. The Kopferman cubes. (Pattern $W$ is only rarely seen bidimensionally, Pattern $\mathbf{Z}$ more than half the time. From "A Quantitative Approach to Figural 'Goodness' " by J. Hochberg and E. McAlister, 1953, Journal of Experimental Psychology, 46, p. 363. Copyright 1953 by the American Psychological Association. Reprinted by permission.)

tion. For example, to describe Pattern $W$ as a bidimensional pattern the authors listed the length of 16 line segments, 25 angles, and the locus of 10 points of intersection. In contrast, the corresponding numbers for Pattern $Z$ are only 12,17 , and 7. According to this description, Pattern $W$ should be less likely to appear bidimensional (more likely to appear tridimensional) than Pattern $Z$ (assuming that the amounts of information for the two tridimensional cubes are equivalent). Estimates of the likelihood of the alternative percepts were derived from a two-alternative forced-choice task. Each stimulus pattern was presented for $100 \mathrm{~s}$. Tones were presented at random intervals during the presentation period. On the occasion of each tone, the observer reported whether the pattern appeared bidimensional or tridimensional. Hochberg found that the bidimensional appearance was reported for Pattern $W$ for only $1.3 \%$ of the probes, whereas Pattern $\mathbf{Z}$ elicited reports of bidimensionality for $60.0 \%$ of the probes. These results, as well as the responses to Patterns $X$ and $Y$, are taken as evidence for a minimum tendency in the perception of form.

It should be noted at this juncture that Hochberg and McAlister's procedure avowedly did not produce a direct test of the tendency toward informational economy (one form of minimum tendency). This should be apparent from the fact that figural goodness is measured in terms of response frequency (Hochberg \& McAlister, 1953, p. 189). It is assumed that the perceptual system exhibits a preference for economy. The investigators' task is then to devise a measure of perceptual 
economy-in this case, in terms of "amount of information" - that accords with the perceptual system's assumed preference for goodness or simplicity. The investigators thus are actually testing their own measure of economy for its psychophysical adequacy. An alternative strategy would be to assume that one's measure of economy is adequate (or to argue that it is so on intuitive grounds, or on the basis of predictive success and generality) and then to use response frequency to assess whether the visual system actually exhibits a preference for economy. In either case, a prior assumption must be made, either about the visual system's preference for economy or about the adequacy of one's metric of simplicity. The lack of a direct test of minimum tendencies (and of the minimum principle) is a common feature of empirical work in this area.

The measure of informational economy is crucial to any empirical test of the minimum tendency. Although Hochberg and McAlister's procedure for assessing informational economy may seem straightforward, its application in this study raises questions. Ostensibly, the intention is to measure the informational economy of alternative perceptual representations. In actuality, the procedure is applied only to the stimulus patterns. This may seem inconsequential inasmuch as the bidimensional appearances of the four patterns may be assumed to have the same properties as the patterns and no others. Thus, when the information needed to describe the drawing is listed, it is plausible to assume that the information needed to describe its appearance as a bidimensional form has also been specified. However, no application to the drawing can measure the information when the perceptual representation is of a tridimensional form, because by definition the latter is characterized by properties that are absent in the drawing (e.g., relative orientation of the faces and internal depth). In fact, the procedure, applied to the drawings, in principle cannot uncover any differences among the tridimensional representations of the patterns. Inasmuch as information is measured simply by counting lines, angles, and intersections in the stimulus drawings and because all of the drawings are projections of the same posited cube, the procedure will necessarily yield the same measure of information for the respective tridimensional representations of the four patterns. Indeed, for the comparison of the frequency of reports of bidimensionality among patterns to be a valid test of the minimum tendency, the authors must assume that descriptive information is constant for the patterns perceived as tridimensional forms. No account is taken of the fact that the drawings present four phenomenally distinct views of a tridimensional cube, and therefore no assessment is made of the relative simplicity of these distinct percepts.

Although Hochberg and McAlister did not seek to provide an explanation of their finding of a tendency toward perceptual economy, their study of alternating perceptual organizations suggests one conceptualization of the process by which perceptual economy is achieved. Consider the finding that the reports for Pattern $Y$ were almost equally divided between bi- and tridimensional responses. One inference which may be drawn from this result is that the perceptual system operates by generating various perceptual representations compatible with the stimulus, assessing the informational content of each and then selecting the representation which passes the test of economical representation. When, as for Pattern $Y$, the alternatives are equally economical, a selection is made at random. This process model may be appropriate only for patterns that are perceptually multistable, or it may be proposed as a general model, applicable whenever more than one perceptual organization is compatible with proximal stimulation, even if only one of these patterns is normally "selected" for perception.

Buffart, Leeuwenberg, and Restle. Recently, Buffart, Leeuwenberg, and Restle (1981) applied the "law of simplicity" or "minimum principle" in the analysis of pattern perception. Their approach shows a kinship with that of Hochberg and McAlister (1953) in its use of feature counting to measure simplicity. However, Buffart et al. (1981) committed themselves to going beyond the psychophysical investigation of minimum tendencies to investigate the minimum principle as a cardinal explanatory principle in the perception of form.

Buffart, Leeuwenberg, and Restle (1981) examined the perceptual tendency, remarked 
earlier by Gestalt psychology, of the percipient to complete a figure, part of which is occluded by another. Buffart et al. designed 25 patterns, each consisting of a square and one or more additional bidimensional figures. Four examples are shown in Figure 2. For each pattern, the subject was asked to trace the contour of the figure or figures that accompanied the square and, for those cases in which the square overlapped the other figure, to be especially accurate in drawing angles (if any) hidden behind the square. The questions of interest were: (a) When will the subject report a completion in contrast to a nonoverlapping mosaic? (b) What completion will be made? Buffart et al. argued that the answer to both questions is provided by Leeuwenberg's (1967, 1971, 1978) coding theory and the minimum principle.

Coding theorists are committed to the idea that perception is a matter of interpretation. Each interpretation corresponds to a "primitive code," which is a set of symbols describing the form of an object within the language

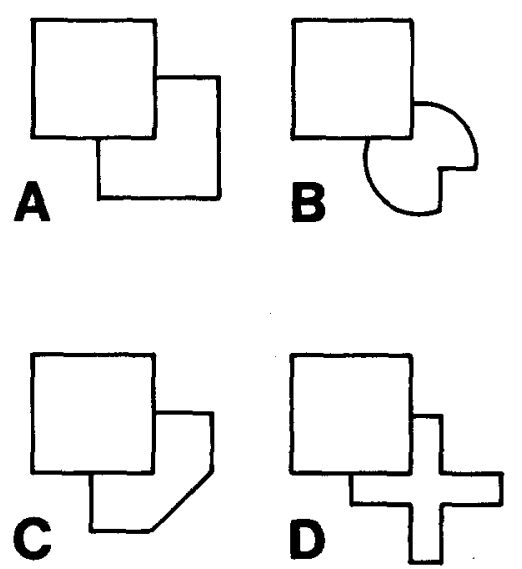

Figure 2. Four of the 25 figures used in the figural completion studies of Buffart, Leeuwenberg, and Restle (1981). (Information loads as computed by the investigators favored a completion for $\mathrm{A}$ and $\mathrm{C}$, a mosaic for $\mathrm{D}$, and assigned equal loads to each interpretation for $\mathrm{B}$. $A$ and $C$ were chosen as completions by 30 out of 30 adult subjects; $\mathrm{D}$ was always given a mosaic interpretation. B was interpreted as a mosaic by 5 out of 30 subjects, receiving a completion by the other 25 subjects. From "Coding Theory of Visual Pattern Completion" by $\mathrm{H}$. Buffart, E. Leeuwenberg, and F. Restle, 1981, Journal of Experimental Psychology: Human Perception and Performance, 7, pp. 242-243. Copyright 1981 by the American Psychological Association. Reprinted by permission.) of coding theory. These symbols are related to various perceptual configurations according to code semantics, which provide rules for constructing a primitive code that symbolizes the relative placement of lines and angles needed to specify a given form. The idea of simplicity is introduced in accordance with various syntactic operations on these primitive codes. The codes are first "reduced" to simplest form via a set of formal operations on the code symbols (e.g., conventional operators to indicate iteration of symmetry). Relative simplicity among alternative codes for the same ambiguous display is then calculated by comparing the number of independent parameters within the code needed to specify fully each perceptual "interpretation." In the words of the authors:

Coding theory consists of the idea that a given display may result in any of several interpretations or codes, that a primitive code can be reduced to a shorter form, that each such code has an information load that consists of the number of independent parameters it uses, and the hypothesis that the perceptual system tends to use the code with minimum information load, according to the law of simplicity or minimum principle.

The law of simplicity, within coding theory, is this: The perceptual system reduces information load and under ideal conditions will arrive at the interpretation having the lowest information load. This, in a natural sense, is the interpretation having the simplest code and can therefore be thought of as the simplest interpretation. (Buffart et al., 1981, pp. 250-251)

As an example of the operation of coding theory, consider Figure 3. The upper portion shows two interpretations of one of the experimental figures (our Figure 2A). Figure $3 \mathrm{~A}$ is a "figural completion"; $3 \mathrm{~B}$ is a "mosaic" interpretation. As defined within code semantics, $\alpha$ stands for a right angle, and $\lambda$ is a unit line segment. To derive a primitive code, one stipulates a beginning point and direction, and then lists the line segments and angles that make up the figure. The primitive code for Figure $3 \mathrm{~A}$, derived by following the path indicated in the figure, is $\lambda \alpha \lambda \lambda \alpha \lambda \lambda \alpha \lambda \lambda \alpha \lambda \alpha \lambda \alpha \lambda \lambda \alpha \lambda \lambda \alpha \lambda \lambda \alpha \lambda$. By counting the number of symbols, one determines the information load; in this case $I=25$. By taking advantage of the repeating or iterated elements, such as $\lambda \alpha \lambda$, the primitive code may be reduced to the following expression: $4^{*}[\lambda \alpha \lambda] 2^{*}[\alpha \lambda] 3^{*}[\lambda \alpha \lambda]$, for which $I=11$ (counting only the iteration numerals and the 

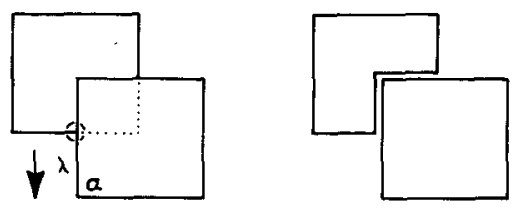

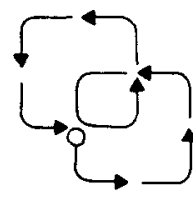

A

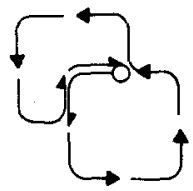

B
Figure 3. Two possible interpretations of Figure 2A. (The upper figures indicate completion (A) and mosaic (B) interpretations. The lower figures show coding paths. The circles indicate where coding begins, and the arrows indicate the direction in which coding proceeds. The letters $\alpha$ and $\lambda$ indicate a right angle and $a$ unit line segment. The primitive code is constructed by listing the elements $\alpha$ and $\lambda$ in order as one proceeds along the coding path. From "Coding Theory of Visual Pattern Completion" by H. Buffart, E. Leeuwenberg, and F. Restle, 1981, Journal of Experimental Psychology: Human Perception and Performance, 7, p. 246. Copyright 1981 by the American Psychological Association. Reprinted by permission.)

elements that stand for angles or line segments; neither the reducing operation itself nor the "conventional"-nonreferringsymbols are counted in the information load of a reduced code). Further syntactic operations of a conventional (and intricate) nature reduce the information load to four. For Figure 3B, the primitive code has $I=26$ and may be reduced to $I=6$. The completion interpretation is therefore predicted. (It may be noted that even though the lower square in Figure $3 \mathrm{~A}$ is perceived as being behind the upper square, the code proceeds from square to square without registering a change in depth, thereby treating the squares as coplanar.)

Buffart et al. (1981) evaluated the information load of the reduced code for the completion and mosaic interpretations. Of the 25 figures, 16 had completions with lower information loads than the mosaic interpretation, 7 had equal information loads, and 2 had more economical mosaic interpretations. For the first set, it was found that $96 \%$ of the subjects produced completions; when the information loads were equal, $45 \%$ of the subjects produced completions; and for the latter set, only $10 \%$ of the subjects produced completions. Comparable results were found with subjects who were graduate students and researchers and with secondary school students. Buffart et al. claimed complete success for their combination of coding theory and minimum principle.

As in the case of Hochberg and McAlister's (1953) study, Buffart et al. strictly speaking have not made a direct test of the minimum principle. They have shown that, on the basis of their coding theory and their posited measure of minimum information load, they can predict subjects' responses. Yet at least two concerns are conflated in this test: (a) whether the perceptual system operates in accordance with a minimum principle, and (b) if it does, whether this principle is mirrored in the formal apparatus of Buffart et al. There can, however, be no independent test of (a). The formal apparatus mentioned in (b), together with the intuitions of past and present experimenters, must serve as the test of (a) at the same time that (b) itself is tested. This notuncommon situation in the logic of experimentation is by no means fatal, and it may be met by application of converging operations and by the long-run empirical fruitfulness of a hypothesis. However, the situation is disconcerting when competing explanations for the empirical outcome are available. Buffart et al. did in fact compare their explanation with other accounts of figural completion, including both the familiarity of the completed figure and a reliance on local cues for overlap, and they claimed relative superiority for their theory. Their case is compelling for the comparison with "local-cue" theory. According to a local-cue approach, a local feature such as a $T$ signals overlap. In agreement with the earlier findings of Donnerstein and Wertheimer (1957), Buffart et al. found that the $T$ subpattern may be associated with a completion or a mosaic depending on the global context. (In Figure 2A the $\mathrm{Ts}$ are associated with overlap and hence completion; in Figure 2D they are not.) It is more difficult to evaluate the comparative claim of the familiarity account, because no measure of familiarity nor any actual tests of familiarity 
are provided. Appeal is made to intuitions, which clearly can vary, because the authors cite a square with one corner lopped off as a case of an unfamiliar figure (the "completion" interpretation of Figure 2C).

A review of the computations required by the coding theory to arrive at the informational loads of the alternative constructions reveals a long serial routine. Although this may be an apt characterization of the calculations required of the investigator, Buffart et al. intentionally left open the question of whether this serial process is carried out by the perceptual system. In fact, they offered assurance that "theoretical work on the process of coding is in progress and does not build on the hypothesis that the process is primarily one of generating a code item by item" (Buffart et al., 1981, p. 272). Nonetheless, they are committed to the notion that the visual system interprets stimulus displays in terms of primitive codes that embody features akin to those found in coding theory, and that these are, by some nonserial process as yet unspecified, reduced to simplest form and compared for informational load.

In a subsequent discussion, Buffart, Leeuwenberg, and Restle (1983) have compared the perceptual process to one of hypothesis testing in the tradition of Gregory (1974). They assumed that a registered pattern of stimulation induces the projection of several perceptual hypotheses (potential interpretations), out of which the simplest (as determined by information load) is chosen for verification against "sensory constraints" (i.e., against a more thorough checking of registered stimulation). During the verification procedure additional hypotheses may be generated, among which the simplest will again be chosen for further verification (Buffart et al., 1983, p. 996). Hence, these coding theorists have proposed that perceptual hypotheses are generated, selected for minimum-information load, and tested for accuracy, all within an internal coding system.

Attneave and Frost. Vickers (1971) proposed that the impression of depth elicited by gradients of optical texture is due to the operation of a principle of informational economy. He contended that economy is achieved by assigning the elements of the texture gradient to that slanted surface on which they would form a uniform (ungraded) texture. On the basis of his observations, Vickers concluded that the minimum principle provides a better account of the operation of texture gradients than either traditional cue theory or Gibson's $(1950,1966)$ direct theory.

Like Vickers, Attneave and Frost (1969) advocated the "minimum principle" as an account of the monocular perception of tridimensional configurations which stands as an alternative to both cue theory and Gibson's theory. Attneave and Frost's formulation of the minimum principle has much in common with Hochberg and McAlister's proposal: "[Monocular] depth perception is determined by tendencies to minimize the variability of angles, lengths, and slopes" (Attneave \& Frost, 1969 , p. 394), with the qualification that the tridimensional organization is "within the set of permissible tridimensional interpretations of the [optical] projection" (p. 391). The notion of "permissible" interpretations is treated by assuming that the rules of perspective are implicit in an analog medium representing physical space (p. 395), within which perceptual representations develop.

Attneave and Frost (1969) tested their version of the minimum principle by examining the extent to which subjects perceive monocularly viewed line drawings of parallelepipeds as tridimensional when this organization minimizes the variability among lines, slopes, and angles. Consider two of the three types of display studied by Attneave and Frost (Figure 4). In both cases, the subject monocularly inspected the drawings and aligned a binocularly viewed rod so that the rod appeared to be a colinear extension of one of the edges of the perceived tridimensional parallelepiped; this procedure was repeated for each of the three leading edges of the two drawings in Figure 4, plus a third (intermediate) drawing. In one case the standard drawing was an orthogonal projection of a parallelepiped such that in the drawing the lines representing edges were of equal length and opposite edges were parallel, that is, equal in slope (Condition 1; actually, nine variant drawings were used in each condition). In the other case, the standard drawing was a polar projection of a cube (Condition 3 ). In this drawing, lines representing edges were 

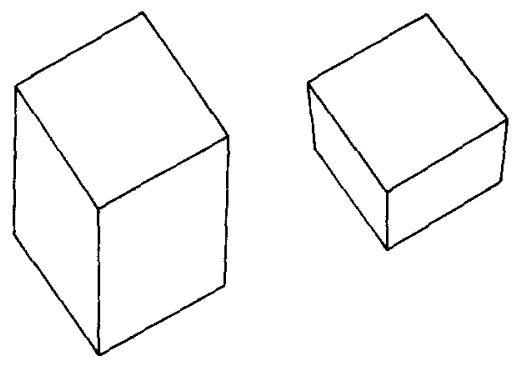

CONDITION 1

CONDITION 3

Figure 4. Illustration of two of the three conditions used by Attneave and Frost (1969, Fig. 2). (In Condition 2 [not pictured], sides were parallel as in Condition 1, but line lengths varied in accordance with perspective. Nine variant drawings [projections from different points of view] were used in each condition. From "The Determination of Perceived Tridimensional Orientation by Minimum Criteria" by F. Attneave and R. Frost, 1969, Perception \& Psychophysics, 6, p. 392. Copyright 1969 by Psychonomic Society, Inc. Reprinted by permission.)

unequal, and the slopes were also unequal, as in a conventional perspective rendering of a cube. This latter drawing affords a projection consistent with viewing a real cube monocularly from a selected reference point. To assess the minimum principle, Attneave and Frost calculated the slant-in-depth of the three leading edges of the hypothetical parallelepiped having the minimum variability among the angles, lengths, and slopes of its edges. They predicted that calculated or hypothetical slant-in-depth would be positively correlated with the subjects' judged slant. More important, the relationship should be stronger in the second case than in the first case. In the second case, conformity with the hypothetical slant-in-depth will equalize the three variables. In the first case, two of the three variables-slope and length-are already equal in the picture plane; taking the figure in depth would make the angles equal but render the lines and slopes unequal. The results reported by Attneave and Frost (1969, p. 394, Figures 3a and 3c) conformed to both of these predictions.

Although Attneave and Frost treated the minimum principle in terms of minimizing variability among the angles, lengths, and slopes of the posited object, Attneave (1972) favored a view that interprets the minimum principle in conjunction with the idea that physical space is represented perceptually as an approximately isotropic analog space. The minimum principle then operates not only to simplify the relationships among the parts of each perceived object (as in the previous case), but also to simplify the relationship between perceived objects and an underlying reference system in this analog medium. Attneave tentatively postulated:

a mechanism that reads momentary tridimensional values of length, slope, angle, and the like out of the spatial representational medium and computes from them an integrated measure of 'goodness' or simplicity that is fed back as a 'hot-cold' signal guiding the tridimensional representation to the simplest state consistent with the constraints of the input, at which the system would achieve stability. (Attneave, 1972, p. 302)

The envisioned mechanism is teleological in the unobjectionable sense that it is guided toward a simple end-state by local decisions along the way. This formulation implies a process that is unlike the model we suggested for Hochberg and McAlister's study. Our interpretation of the Hochberg-McAlister data suggested a process in which the minimum principle acts as a selective rule for choosing among fully formed alternatives, all of which are generated for assessment. In contrast, Attneave's formulation suggested that the minimum principle guides the construction of a single (most economical) representation, consistent with input and the laws of projective geometry. (Attneave, 1972, imputed the latter to his postulated spatial representational medium; see p. 302.) It is as if the evolution of the perceptual representation is directed in progress; the various decisions required as this evolution progresses are resolved by the minimum principle.

Attneave and Frost (1969) contended that their results are more consistent with a version of the minimum principle than with cue theory or direct theory. However, as was the case with Vickers' study, their experiment was not designed to decide among rival accounts, and their assessment of the relative merit of the minimum principle did not depend upon differing empirical predictions derived from the competing accounts. As in the case of Buffart et al. (1981), the characterization of the competing theories is one that adherents of those theories would question. Cue theory is understood as involving 
an item by item list of the relation between proximal lines and angles and possible distal slopes, making no mention of the inference rules or algorithms common to cue theory. Direct theory is characterized as involving a cumbersome cognitive apparatus for computing and solving the higher order variables of that theory, totally at variance with Gibson's (1966) notion of "information pick-up." Indeed, in a subsequent evaluation of this experiment Attneave (1972) remarked: "The foregoing comparisons show, if nothing more, the extraordinary difficulty of experimentally confirming or disconfirming a Prägnanz theory as opposed to its alternatives . . . I doubt at this point that any experiment of the present kind is going to settle the issue in a decisive way" (p. 300).

Perkins. Perkins (1976; see also Perkins \& Cooper, 1980) investigated two issues relating to Prägnanz in perception. First, he sought to show that the imposition of Prägnanz or "good form" in the interpretation of visual stimuli is constrained by (or is in accordance with) the rules of projective geometry (see also Perkins, 1972). Second, he investigated the notion that the attribution of good form to visual stimuli is a "good bet" (i.e., that a Prägnanz assumption would guide the visual system toward accurate perception of form).

The notions of Prägnanz and of geometric constraints used by Perkins (1976) may be illustrated in conjunction with Figure 5. For the present purpose, restrict attention to Angles $L$ and $R$ and potential axis of Symmetry $S$ (as illustrated in Figure 5a and applied to the others), and regard each of the shapes as a tridimensional object. A Prägnanz interpretation would consist in attributing right angles to the corners of this object, or in attributing to the object symmetry about Line S. For Figure $5 \mathrm{~b}$, casual inspection may reveal that three alternative tridimensional organizations are perceptually favored: (a) the left end appears rectangular, with Angle $\mathrm{L}$ perceived as a right angle; (b) the right end appears rectangular, and $R$ is perceived as a right angle; and (c) the object appears symmetrical about Line $S$, with Angles $L$ and $R$ equal to one another. Each of these interpretations is consistent with projective geometry; that is, each of these posited tridimensional objects could project Figure $5 \mathrm{~b}$ in accordance with conventional projective geometry. By contrast, for Figure $5 \mathrm{~g}$ geometric constraints allow a rectangular interpretation of Angle $R$, but do not permit this attribution to Angle $L$, nor is symmetry permitted. For any of the figures, once a given angle is decided, projective geometry can be applied across the rest of the figure to determine all sides and angles of the posited tridimensional object.

Within this framework, Perkins (1976) tested three predictions: (a) perceptions of symmetry or rectangularity will occur more frequently than chance, (b) such percepts will occur more frequently when they are consis-

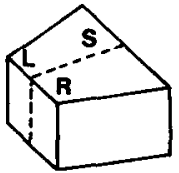

(a)

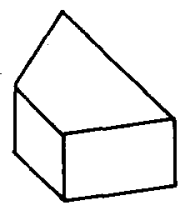

(e)

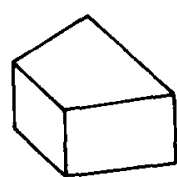

(b)

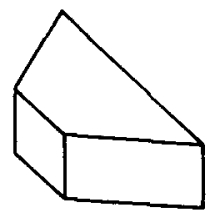

(1)

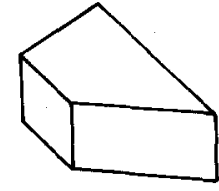

(c)

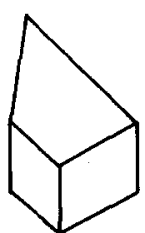

(g)

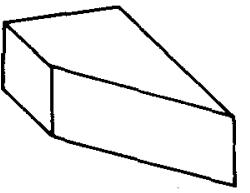

(d)

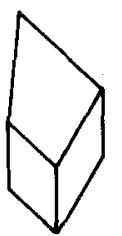

(h)

Figure 5. The stimulus shapes used by Perkins (1976, Fig. 1). (Angles $L$ and $R$ are defined on each shape about the potential axis of symmetry indicated by the dotted line in View a. From "How Good a Bet is a Good Form?" by D. Perkins, 1976, Perception, 5, p. 394. Copyright 1976 by Pion Ltd. Reprinted by permission.) 
tent with projective geometry, and (c) subjects' estimates will approximate the geometrically correct values; that is, when a subject reports rectangularity or symmetry in a shape, the estimates of the nonright angle or the symmetric angles will accurately reflect the value determined via projective geometry. Eight subjects were asked to provide verbal estimates of Angles $L$ and $R$ (to within $5^{\circ}$ ) in Figure $5 \mathrm{~b}-5 \mathrm{~h}$, under instructions to view each drawing as a depiction of a tridimensional object. Although the data did not conform to these predictions in every instance, overall agreement with the predictions was good. Subsequent work by Perkins (1982) tested perceivers' accordance with geometric constraints on a wider variety of stimuli, and indicated that perceivers are flexible, if sloppy, geometers.

Perkins viewed the process of generating individual percepts as a directive one, involving serial application of the Prägnanz hypothesis by the visual system. The Prägnanz assumption is applied to a salient feature of the stimulus figure and is accepted if it is consistent with the constraints of projective geometry. If the Prägnanz attribution (e.g., this corner is right angled) is adopted, then "the implications in terms of shape, size, slant, connectivity ... are propagated to other parts of the scene" (Perkins, 1976, p. 403). The process might be repeated several times until a consistent figure is discovered.

A more detailed analysis of a serial, directive process as envisioned by Perkins has been provided by Simon (1967) and may be illustrated by his analysis of the perception of the Necker Cube:

\section{A. A scanner moves over the stimulus and detects simple configurations. By simple configurations' are meant figures like those the Gestalt psychologists call 'good': straight lines, especially if horizontal or vertical, right angles, squares, circles, closed symmetrical forms.}

B. When a simple configuration has been detected, the scanner proceeds from it to the other elements of the stimulus, providing them with simple interpretations in relation to the initially discovered simple configuration. This process continues until an internal representation has been constructed for the entire stimulus, or a contradiction has been encountered.

C. If a contradiction is encountered, the interpretation is rejected and step $A$ is repeated-usually with a different initial position of the scanner. (Simon, 1967, p. 4)
A Gestalt theorist would be appalled by Simon's process model. The serial, part by part examination of features, and the incremental trial-and-check build up of the figure is as remote as could be imagined from the Gestalt approach. (See Koffka's, 1930, pp. 162-168, detailed analysis of the Necker Cube.) Nevertheless, in implying (Point B) that what is taken as a simple interpretation of any one part will condition the interpretation of other parts Simon adopted a holistic approach that the Gestaltist might find agreeable. This point in Simon's exposition requires clarification. It implies that interpretation of one element constrains the interpretation of the other elements, thereby helping to determine what will be accepted as a simple interpretation. This relation of constraint would seem to reflect some unspecified knowledge structure that must be attributed to the visual system, and that presumably reflects Perkins's (or Attneave's) geometric constraints.

The directive process model just sketched addresses the how of Prägnanz. Perkins (1976) answered the question of why by viewing Prägnanz as a working assumption adopted by the percipient as a result of commerce with the environment, in which the perceptual system learns that symmetry and figural goodness are good bets. Assessment of this claim about the environment would involve considerable "ecological sampling" (Brunswik, 1956) on the part of the investigator. Second, the perceptual system uses past experience (prior frequency) to decide between equally possible Prägnanz interpretations (e.g., rectangular and equilateral interpretations of the projection of a parallelepiped). It should be noted that Prägnanz, in Perkins's usage, departs drastically from the Gestalt formulation and operates as a favored perceptual hypothesis or schema in a manner compatible with the type of hypothesis-testing theory favored by Gregory (1974).

Perkins's treatment of Prägnanz as hypothesis or "good bet" raises a troublesome question regarding the relationship between a minimum principle and what may be called the likelihood principle. The likelihood principle, considered by Helmholtz as the fundamental rule of perceiving, is the claim that 
the perceptual system constructs a representation which is the most likely interpretation given the history of exposure to proximaldistal correlations (through learning or evolution). Often the most economical percept seems intuitively also to be the most likely percept.

As illustration, consider the Distorted Room phenomena. In one version of this well-known Ames demonstration, a room constructed with trapezoidal floor, walls, and ceiling is viewed monocularly from a position such that the resulting retinal image is (ideally) equivalent to the image that would be associated with a conventional rectangular room viewed from that position. Under these conditions, the room appears to be rectangular, and the perception persists even when persons take up positions in opposite corners of the room, with the result that the persons appear to be of grossly different sizes. The rectangular appearance of the room is consistent with a minimum principle inasmuch as more information would be required to define a perceptual representation of a Distorted Room (e.g., the angles formed by each of the four corners are unique and the sizes of the windows differ). However, the standard explanation of the appearance of the Distorted Room makes no mention of a minimum principle. The conventional explanation of these findings, which stresses the assumptions that the observer brings to the situation, amounts to an application of a likelihood principle: It is more likely that the given retinal image was caused by a rectangular room than by a distorted room. Granting the claims of the rival accounts, we have a case of correspondence between the simplest and most likely perceptual representation. Similar remarks apply to a number of Ames' other demonstrations (e.g., the Ames Chair).

The kinetic depth effect, a widely cited instance of the perception of depth through motion (Braunstein, 1976; Ullman, 1979), provides another illustration of the congruence between the economical and the likely. When a rotating wire-frame object is illuminated by a point source of light behind a screen (Figure 6), the shadow cast upon the screen might be perceived as a two-dimensional object changing its shape or as a stable three-

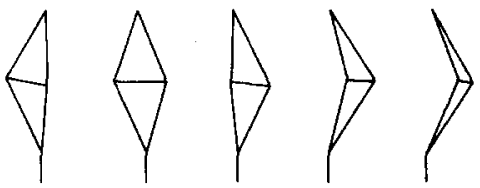

Figure 6. Successive views of a rotating wire object projected onto a screen. (From Introduction to Perception [p. 117] by I. Rock, 1975, New York: Macmillan. Copyright 1975 by Macmillan. Reprinted by permission.)

dimensional object rotating about a central axis. Clearly, a minimum principle would predict that the three-dimensional configuration would be perceived, rather than a twodimensional configuration whose component line segments are constantly altering their lengths and angular relations. In fact, the observer does report perceiving a rigid 3dimensional configuration rotating in depth. Here again, as with the Distorted Room, one might have forecast the outcome by drawing on the rule that the perceptual system is disinclined to posit unlikely objects, in this case an object that continually undergoes covariations of length and angle. (Evidence purporting to support this interpretation has been presented by Rock \& Smith, 1981). Once more there seems to be correspondence between the most economical percept and the most likely percept.

Marr. Marr (1982) and his co-workers have developed a broad theoretical approach to vision that touches on two aspects of perceptual economy: (a) In its description of the structure of the environment it explicitly relies on assumptions that can be characterized as Prägnanz-like, and (b) it provides an interesting conception of a minimization process.

Marr and his colleagues have enlisted the powerful resources of artificial intelligence techniques to create a machine model that simulates the human visual system. Their approach consciously seeks to constrain the many possibilities for creating machine "vision" programs by taking into account the findings of neurophysiology and psychophysics. The primary focus of the work discussed by Marr (1982) is "early vision," which is regarded as the generation of a perceptual representation prior to such cognitive activi- 
ties as object recognition (cf. Marr, 1982, pp. 268-269; Marr \& Nishihara, 1978). The theory proceeds against the explicit background that the purpose or biological function of early vision is to engender a representation of the distal scene, including the shape, orientation, texture, and color of surfaces. At the core of this approach is the idea that the visual system has been engineered to embody certain "assumptions" about the physical properties of the environment, which underlie the processes that extract information about the environment from retinal stimulation.

Although Marr (1982) presented his theory independently of any explicit discussion of Prägnanz, the assumptions that are assigned to the visual system generally attribute Prägnanz-like qualities to the environment. In the first assumption, the Prägnanz quality of smoothness is conjoined with elaborateness of articulation: "The visible world can be regarded as being composed of smooth surfaces having reflectance functions whose spatial structure may be elaborate" (Marr, 1982, p. 44). A later assumption attributes homogeneity within various scales of this elaborate structure: "The items generated on a given surface by a reflectance-generating process acting at a given scale tend to be more similar to one another in their size, local contrast, color, and spatial organization than to other items on that surface" (Marr, 1982, p. 47). A further assumption seeks object boundaries on the basis of common fate: "If direction of motion is ever discontinuous at more than one point-along a line, for example-then an object boundary is present" (Marr, 1982, p. 51). Finally, in the case of stereopsis it is assumed that "disparity varies smoothly almost everywhere" (Marr, 1982, p. 114). From these basic assumptions and others, Marr and his co-workers have developed accounts of edge detection (Marr \& Hildreth, 1980), stereopsis (Grimson, 1981; Marr, 1982; Marr \& Poggio, 1976, 1979) and of the perception of motion (Ullman, 1979), shape (Grimson, 1981; Horn, 1977; Ikeuchi \& Horn, 1981), surface texture, brightness, lightness, and color (Marr, 1982). Interestingly, these Prägnanz assumptions are imputed to the visual system as assumptions about the actual structure of the physical environment. If Marr's theory is correct, the operation of the visual system does indeed depend on the assumption that the world has Prägnanz qualities (i.e., that objects in the typical environment have physical properties that are characterized by smooth variation, homogeneity, and expanses of rigid structure).

Marr's work has provided an interesting conception of the minimization process. Marr and Poggio (1976), drawing on Julesz (1971, 1974), developed a variant of the directive process models previously discussed. Julesz proposed a "cooperative" model of global stereopsis (i.e., of the process by which surfaces-in-depth are determined from binocular disparity among local features). A characteristic of cooperative models is that they depend on numerous local interactions in parallel that "cooperate" to achieve a result. Marr and Poggio implemented a cooperative algorithm that computed surfaces-in-depth from disparity, through iterative operations on local disparities. The operation of the algorithm incorporates the assumptions of continuity or smoothness and uniqueness (i.e., a feature from one image is matched with only one feature from the other image). The algorithm is "computed" by iterative local interactions among "cells" that respond to local disparities, and that are connected to other "cells" in their neighborhood through either excitatory or inhibitory connections (by adjusting the excitatory and inhibitory connections, one can realize various distinct algorithms).

An iterative, cooperative algorithm, realized by a net of "cells," provides an example of a directive, bottom-up process that iteratively converges on surfaces-in-depth possessing the Prägnanz quality of smoothness. Although Marr and Poggio (1979) subsequently abandoned this algorithm for one that does not depend on cooperativeness in its fundamental operation (it rather compares spatial frequency filterings of monocular input from each eye), their new model does use a cooperative process for resolving ambiguities (Marr, 1982). In general, cooperative processes provide a means for envisioning a directive process that converges on smooth forms working bottom-up from many local inputs.

\section{Perception of Movement}

Restle. We conclude our presentation of illustrative applications with three studies of 
the perception of motion. The first is provided by Restle's (1979) reanalysis of Johansson's (1950) widely cited experiments.

Restle applied a line of analysis similar to that of Buffart et al. (1981) previously discussed and draws on Leeuwenberg's (1978) coding theory. As with Buffart et al., perception is viewed as the genesis of an interpretation, in this case of the motion of dots on an oscilloscope. The motion patterns can give rise to various groupings of the dots, depending upon how common and relative motions are allotted (cf. Duncker, 1929). Each pattern can give rise to two or more interpretations (see Figure 7). These are described in codes which are reduced to simplest terms and compared for information load. In Restle's words:

Different interpretations, when fully reduced, may end up with different information loads. The theory states that the observer then will perceive the simplest interpretation, that is, the interpretation with the minimum information load (Hochberg \& McAlister, 1953). If two or more interpretations have equal information load, then the display is ambiguous in practice, and either or both interpretations may be seen. (Restle, 1979, p. 2)

As noted earlier, the general approach is similar to the one introduced by Hochberg and McAlister (1953) in that the codes are essentially lists of the features that have to be specified to describe a particular perceptual representation. Over a large and diverse set of motion configurations, Restle found a high degree of agreement between the interpretations that were actually perceived and the ranking of the various possible interpretations with respect to their informational loads as computed by the investigators.

Restle mentioned briefly two mechanisms by which a perceptual system could arrive at the minimum information load. One is by generating interpretations in nonsystematic order and settling for the intepretation that is most economical. This is an example of the "selective" process discussed in conjunction with Buffart et al. (1981) and Hochberg and McAlister (1953). Restle was wary of this hypothesized process because of the cumbersome number of serial computations that must be performed and the difficulty of devising a means for determining the order in which the candidate interpretations will be generated and tested. As an alternative, Restle

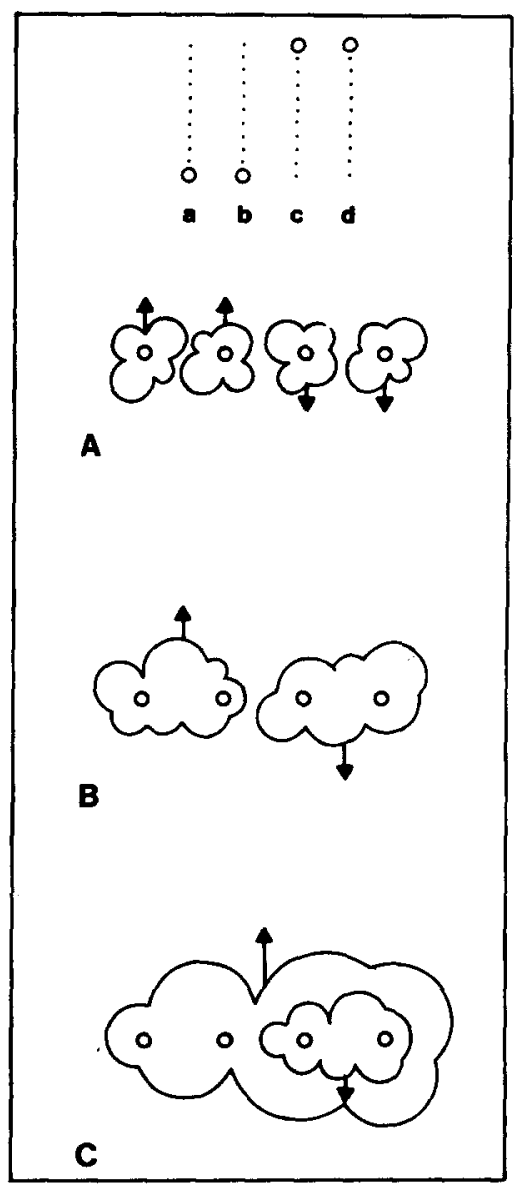

Figure 7. A motion pattern (top) and three interpretations. (Dots $a$ and $b$ move upward, while Dots $c$ and $d$ move downward; velocity is equal for all dots and varies as a sine function [with highest velocity at the center of the motion path]. In Interpretation $A$, the four motions are perceived as independent. In Interpretation $B$, the dots are perceived as two independent pairs. In Interpretation $\mathrm{C}$, the whole system is perceived as moving upward, with a subunit [Dots $\mathrm{c}$ and $\mathrm{d}$ ] moving downward relative to the other subunit [Dots $a$ and b]. Interpretation $C$ could be reversed, so that the whole system moves downward and Dots $a$ and $b$ move upward relative to Dots $c$ and $d$. The information loads for the three interpretations, as calculated by Restle (1979), decrease from A to B to C. Of Johansson's 14 subjects, 13 definitely reported seeing two pairs of dots, and 11 of these reported the motions as a single system with two subunits. From "Coding Theory of the Perception of Motion Configurations" by F. Restle, 1979, Psychological Review, 86, p. 7. Copyright 1979 by the American Psychological Association. Reprinted by permission.)

cautiously toyed with the idea of a mechanism in the spirit of that favored by the Gestalt theorists (e.g., Köhler, 1920): 
It is even possible that the process of finding the minimum information load may resemble physical processes described by thermodynamics-though physical systems reliably find minimum energy states, any calculations are performed not by the system under study but by the scientist. This may be just as true in the analysis of perception. (Restle, 1979, p. 23)

If this analogy with thermodynamics is followed closely, here is an example of a "directive" process in which the minimal "solution" is found by the momentary interaction of a large number of microprocesses. An economical percept is realized as a stable state of the perceptual system, which is achieved at a minimum energy level. According to this conception, the features of the perceptual representation are presumed to be directly related to the characteristics of a distributed process in the nervous system (e.g., an economical motion path is "computed" by a distributed neural process achieving equilibrium at a minimum energy level). Restle regarded it as an advantage of this tentative, qualitative account that it might achieve the results described by coding theory without engaging in the cumbersome serial calculations that must be performed by the investigator in applying the codes.

A third reading of Restle's analysis is that economy of representation resides exclusively in the scientist's description of the percept in accordance with a simplicity metric, rather than in the physico-chemical or psychological processes that generate the percept. This formulation is suggested by the fact that in common with earlier mentioned investigators, Restle made no effort to consider the processing load implied by the various codes. For example, the common motion (direction and velocity) of a set of points is said to have a smaller informational load than the same number of points interpreted as independently moving. This is indisputably true if the claim is confined to the descriptive codes required by the two alternatives taken as givens. However, the economy is achieved by imputing to the visual system what may not be available to it as information except as the product of considerable processing (i.e., that the points have a common fate). Thus, when the processing demands of the rival perceptual interpretations are compared, they may not differ despite the fact that investigators can offer descriptive codes of the final percept which differ in informational content. Economy of form as measured by a formal metric may not correspond to economy of process. In such a case, any tendency of the perceptual system toward the perception of simple configurations would have to be explained on grounds other than economy of information load as determined within coding theory.

Ullman. Working in the tradition of Marr (1982), Ullman (1979) developed a model of motion perception that relies on a "minimal mapping" principle. Ullman divided the problem of motion perception into two parts: the correspondence problem and the threedimensional interpretation problem. The first is the problem of matching the various elements within temporarily separate "frames" of retinal stimulation (either separate time slices of continuous motion or successive presentations in apparent motion). On the basis of empirical study of apparent motion, Ullman concluded that correspondence occurs among "elements" or "tokens" within the image (rather than among entire object configurations, although the entire configuration may influence the matching). Local correspondences are governed by "affinity" among tokens, which is determined by a similarity metric that includes the spatial proximity, brightness, orientation, and length of the tokens (see Figure 8). The problem is then to derive a globally consistent set of matches

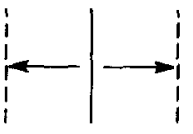

A

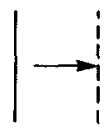

B
Figure 8. The effect of distance on the affinity between line segments. (The solid lines indicate the first frames; the dashed lines, the second; the actual stimulus consisted of solid line segments in both cases [presentation time $120 \mathrm{~ms}$ and ISI $40 \mathrm{~ms}$; monocular viewing]. For Pattern $A$, in which the second frame contains two line segments equally distant from the first and the same in other respects, the correspondence function yields a one-many mapping [two concurrent motions are perceived]. When one of the two distances is now increased as in Pattern $B$, the likelihood of only one motion increases, until a distance is reached at which only the one motion is perceived. From The Interpretation of Visual Motion (p. 36] by S. Ullman, 1979, Cambridge, MA: MIT. Copyright 1979 by MIT. Reprinted by permission.) 
based on the competing affinities among tokens in successive "frames" or "snapshots," in such a way that every element in each frame is matched with at least one element in the other frame. Ullman contended that this matching process is a low-level, autonomous process that generally proceeds independently of semantic interpretation.

Ullman proposed that matching is achieved by computing the "minimal mapping" among elements. In effect, the minimal mapping is the one that minimizes the sum of the distances traveled between frames among the matched elements. Ullman suggested that this minimizing computation can be achieved by a large number of local, simple processing units operating in parallel and interacting. These provide a computational architecture for solving a minimizing function through local, iterative interactions from the bottom up. (Ullman used a modified Lagrangian function, a relative of the equations used to describe some of the thermodynamic processes to which Restle alluded.) Although
Ullman did not develop a fully implemented model, his characterization of the computational process provided an interesting conception of a bottom-up directive process and is akin to a growing number of proposed parallel computational architectures (Feldman \& Ballard, 1982; Hinton \& Anderson, 1981; Uhr, 1982).

Cutting and Proffitt. Wheel-generated motions have been the focus of the theoretical analysis of organizational principles in motion perception (e.g., Borjessen \& von Hofsten, 1975; Cutting \& Proffitt, 1982; Duncker, 1929; Hochberg, 1957; Johansson, 1973; Wallach, 1965). These motions consist of two or more luminous points placed anywhere from the center to the rim of a rolling wheel (see Figure 9). The trajectory of each of these elements through a fixed set of spatial coordinates defines its absolute motion. The absolute motions of a given set of elements usually are analyzed perceptually into the common motion of the whole configuration (e.g., the horizontal motion of a wheel rolling
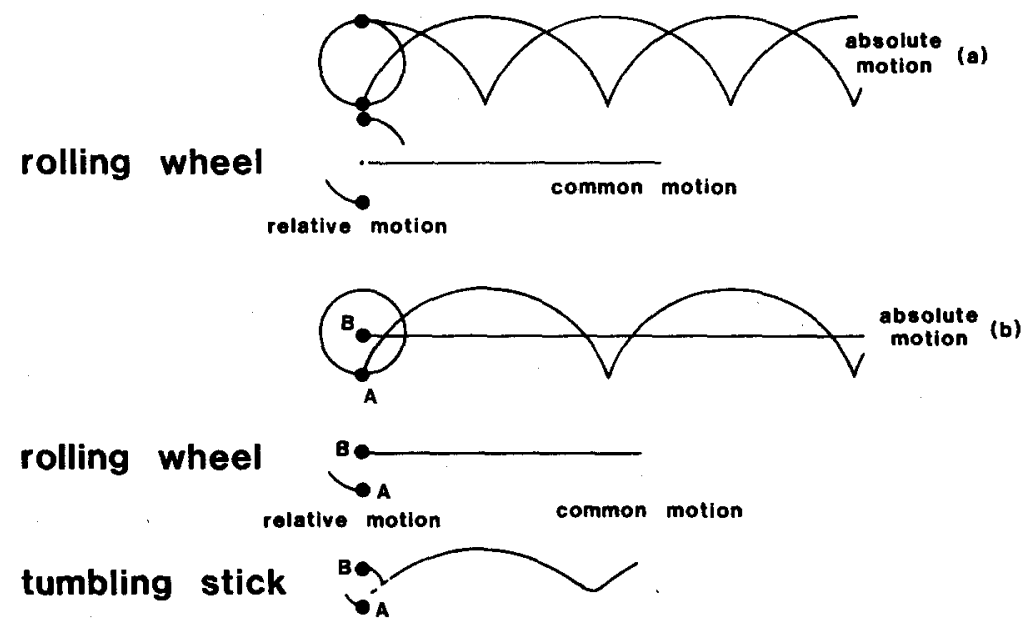

Figure 9. Two stimuli used as prototypes to describe wheel-generated motion. (a. A two-light stimulus with lights mounted $180^{\circ}$ opposite from one another on an unseen wheel rim. The absolute motion paths are two cycloids, $180^{\circ}$ out of phase. The relative motion paths, in contrast, are circular and $180^{\circ}$ out of phase around their midpoint. Common motion is the path of this midpoint, which is linear. b. A twolight stimulus with one light on the perimeter, and one at the center. Absolute motion paths are a straight line and a cycloid. The relative and common motion paths, however, depend on the particular version of the object seen either as a rolling wheel or as a tumbling stick. In the rolling-wheel version, relative motion occurs only for Light A, rotating about Light B. Common motion occurs for both and is linear. In the tumbling-stick version, both lights have relative motions, rotating $180^{\circ}$ out of phase around their midpoint, and they both have common motion, describing a prolate cycloid. From "The Minimum Principle and the Perception of Absolute, Common, and Relative Motions" by J. Cutting and D. R. Proffitt, 1982, Cognitive Psychology, 14, p. 221. Copyright 1982 by Academic Press, Inc. Reprinted by permission.) 
on a flat surface) and the relative motion of each element to other configural elements (e.g., a point on the rim revolving about a point at the center of the wheel). On the assumption that "geometric constraints" as discussed previously are in place, the absolute motions of any set of elements may be analyzed in principle into any of a potentially infinite set of combinations of common and relative motions. It is the widespread view that the visual system settles on a particular combination of common and relative motions through the operation of a minimum principle.

Two separate minimum principles have been proposed to account for the selection of one among the many possible common/relative motion pairs. The first operates in conjunction with the assumption that common motion is the first element to be abstracted from the absolute motions of the stimulus configuration, leaving relative motion to be specified as the residual. A minimum principle is operative insofar as common motion is abstracted in such a way that "the percept entailing the least number of changes is obtained" (Hochberg, 1957, p. 82). In the case of a wheel with a luminous point at the center and on the perimeter rolling along a flat surface, this principle predicts the perception of a horizontally moving center with a satellite element revolving about it, rather than various other allotments of common and relative motion in which the center of rotation bobs up and down. The minimum principle operates to minimize common motion as a straight line.

According to the rival account, relative motion is abstracted first in such a way that the sum of the momentary relative motions equals zero. This entails that the points rotate about their centroid. In the case of a wheel with one light at the center and one on the rim, this principle implies that the two lights are perceived as opposite ends of a tumbling stick whose center of rotation describes a prolate cycloid (see Figure 9). Although previous authors had urged either that common motion or that relative motion is abstracted first, calling upon one or the other of the minimum principles, Cutting and Proffitt proposed a third view: that minimization processes operate on both common and rel- ative motions simultaneously and that the process which is completed first determines the percept. The first motion component to be minimized is extracted from the event, and the second motion is treated as the residual.

The work of Cutting and Proffitt (1982) raises an important question concerning the notion that perception is broadly guided by a minimum principle. These authors referred to their work as one example of a general rule that perception tends toward simplicity, not only in motion perception but in pattern perception and the perception of ambiguous figures as well. At the same time, these authors were careful in the case of motion perception to distinguish between the two different minimum principles mentioned previously. These are cast as distinct minimum principles presumably because they involve the minimization of quite distinct quantities or attributes. In what sense may they both be said to instantiate the operation of a single Minimum Principle? Although one may reply on intuitive grounds that these principles and the others we have discussed are linked under the common notion of economy or simplicity, in the absence of a commonly applicable metric of simplicity it is difficult to make a case that they are all manifestations of a single, formulable principle of perceptual processing.

This question as well as others pertaining to the idea that the minimum principle can be used as a broad explanatory principle in perceptual theory are addressed in the following section.

\section{Evaluation of the Status of Minimum Tendencies and Minimum Principles in Theories of Perception}

Our selection of experimental illustrations reveals that minimum tendencies are not merely another exhibit in the arcade of perceptual curiosities. Nor have the suggested applications of the minimum principle been restricted to a narrow band of phenomena. It has been regarded by some theorists (Attneave \& Frost, 1969; Hochberg, 1964) as a core explanatory principle in perception; others (e.g., Hochberg, 1974; Perkins, 1976) have explained the minimum tendency via likelihood. 
In preparation for evaluating the minimum principle and other proposed explanations for minimum tendencies, we address two questions regarding the idea of perceptual economy. The first pertains to the relation between the presumed preference for percepts that satisfy a minimum principle and the seeming obligation of the percept to adhere to the "geometric constraints" of optical stimulation. The second question pertains to the theoretical implications of adopting one or another metric of simplicity in evaluating the "minimal" properties of stimulus and percept.

\section{Minimum Principle and Geometric Constraints}

Whatever the ultimate status of the minimum principle, clearly such a principle must operate within the constraints imposed by proximal stimulation. The need to take into account constraints imposed by stimulation is obvious, for otherwise the minimum principle could not be expected to culminate in perceptual representations that are signifcantly correlated with the environment. (In the absence of constraints, the principle merely predicts a relative preponderance of percepts classified as "simple" by some metric; indeed, in the absence of constraints, areas imaged next to one another on the retina need not be contiguous in the experienced visual field.) However, the notion of constraints provided by proximal stimulation itself needs further clarification.

A number of the authors discussed in the preceding section explicitly construed the minimum principle as operating within the constraints of projective geometry. It is a matter of empirical fact that the perceptual representations generated in response to a given pattern of proximal stimulation fall (at least roughly) within such constraints: A circular pattern on the retina gives rise to the representation of any of a family of ellipses (depending upon the perceived slant), but not to the representation of a square. Yet the laws of projection relating distal configurations and their retinal projections are not themselves given in the pattern of stimulation. Indeed, the laws of geometric optics that apply to the light stimulating the retinas are one thing; the physiological and psychological processes that are initiated by stimulation of the retinas are another. The means by which the perceptual system generates representations that are more or less in accordance with projective geometry must be sought within the perceptual system itself, as psychological rules or mechanisms. A clear distinction must be maintained between projective geometry per se, and "geometric constraints" regarded as rules of the perceptual process. Such constraints include the familiar relation in which projective shape limits the permissible range of perceived shape-at-a-slant, and in which visual angle limits the permissible range of perceived size-at-a-distance.

Given that geometric constraints are in place, what would be left in perception for the minimum principle to accomplish? The minimum principle would operate within these constraints by determining which member of the range of perceptual representations compatible with stimulation will be experienced. More specifically, if a minimum principle is to operate, two conditions must be met. First, there must be a range of representations compatible with stimulation for the minimum principle to decide among. Second, the members of this range must differ according to a common metric of simplicity in such a way that there can be defined a simplest, or locally simplest, representation.

For the first condition to be met, registered stimulation, together with the psychologically real geometric constraints, must be insufficient to determine a percept. Whether this is the case may be a subtle question, because it may be difficult to draw an absolute boundary between those mechanisms that enforce geometric constraints and further processing mechanisms. A Gibsonian might contend that in most cases the constraints placed upon permissible perceptual representations by proximal stimulation are sufficient to determine a percept, so that the minimum principle must be regarded as a default rule operating in those few cases of impoverished stimulation. However, it should be noted that if our argument about geometric constraints is correct, this Gibsonian contention cannot be justified solely on the basis of ecological optics (Gibson, 1966, 1979). (Our argument does not depend on adopting the conventional 
premise that stimulation is inherently equivocal from a geometrical point of view.) Even in a Gibsonian account of perception, mechanisms are required to "pick up" or "detect" the information available proximally. It seems unlikely that an a priori argument could be formulated to preclude the operation of a minimum principle within these detection mechanisms. Even if proximal stimulation is unequivocal with respect to distal properties from the point of view of ecological optics, this does not entail that the mechanisms that determine the percept need fall under our category of geometric constraints; the psychologically real detection mechanism might use geometric constraints to determine a range of percepts, with a minimum principle taking up the slack. In any event, whether our first condition obtains or not is a question that can be answered only by an investigation of the actual functioning of the visual system.

The second condition states that the range of representations permissible within the geometric constraints must be comparable on a single metric of simplicity. We may ask whether there are any classes of situations for which this clearly is or is not the case. A clear set of cases meeting our second condition is that in which the proximal stimulus constrains the percept in all but a single dimension or attribute, along which a simplest representation may be defined. We have discussed several cases of this type. In the work of Hochberg and McAlister (1953), Buffart et al. (1981), and Perkins and Cooper (1980) the dimension of variation was "good form." Although a unique minimal representation could not always be defined for the stimulus pattern, the competing economical representations generally were comparable and so resolvable within the individual metrics of simplicity (e.g., by perceptual alternation among two or more representations that are equally or nearly equally "simple" or "good"). Similar remarks apply to Restle's (1979) analysis of motion perception in terms of coding theory.

In contrast, a set of cases in which no single minimal solution (or no set of obviously equivalent minima) presents itself is that in which the proximal stimulus allows for a trade-off along two or more dimensions. Cutting and Proffitt's (1982) study of wheel- generated motions is a case in point. Such motions can involve minimization of common or relative motion. As we mentioned, the metrics provided by Cutting and Proffitt yield no basis for comparing these competing dimensions of minimization with one another, even though the measurement of, say, minimal relative motion in terms of the sum of momentary relative motions provides an unequivocal measure of economy within that dimension. Another example involves the perceptual trade-off between size and distance. Continuous increase or decrease in visual angle is compatible with either an expanding and contracting object at a constant distance or an object of constant size approaching and receding. What would a minimum principle predict in such a situation? Change in either size or distance might be minimized, or a compromise might be reached. When considering a single case in isolation, there seems to be no reason a priori for finding that minimization of change in one attribute is simpler than minimization of change in the other. Resolution of the trade-off in this case would have to be determined empirically. Further explication of the empirically determined operation of the minimum tendency would then involve other principles of perception.

Beyond these two conditions, there is an additional question that arises in applying the notion of geometric constraints, and therefore, in deciding what minimum configurations may be available within such constraints. How much of the environment should be included in setting the constraints within which a minimum configuration may be defined? One might include the spatial layout as sampled by a freely moving organism over a period of a few minutes, the current visual field, or some portion of the latter (these possibilities are not exhaustive). If a minimum tendency or minimum principle is to operate as envisioned by these authors, the segment of the environment must not be too small. For example, the application of a coding analysis to the four dots in Figure 7 depends upon all of them being registered by the perceptual system.

The authors discussed previously fall into two groups on this matter. Those treating the perception of form and depth used static 
figures; here it is expected that the single drawing, perhaps as filling the visual field at any given moment, is the effective stimulus, and its proximal registration sets the geometric constraints within which a minimum (or relative minimum) configuration will be determined. By contrast, the motion studies of Restle (1979), Ullman (1979), and Cutting and Proffitt (1982) obviously demand that a minimum motion configuration be determined over time. The interval between frames determined the time limit in Ullman's studies. Cutting and Proffitt's wheel-like motions were on the screen for $2.5 \mathrm{~s}$; Restle's dots cycled in 1 to $4 \mathrm{~s}$. Neither of these authors discussed the relation between exposure time and the perception of an "economical" motion configuration.

Hochberg $(1974,1982)$ has claimed that the difficulty in setting bounds on the extent of the visual scene that must be included in a minimizing calculation poses a perhaps insurmountable obstacle to any account of perception based upon a minimum principle (and, by implication, to the possibility of measuring a minimum tendency). Hochberg observed that "impossible" figures, such as those in Figure 10, present a difficulty for the application of a minimum principle to the perception of form. For example, an assumption of "figural goodness" leads to viewing the left and right sides of Figure $10 \mathrm{~A}$ as composed of solid rectangular prisms, put together as in a picture frame. Yet the figure as a whole cannot be consistently organized as a picture frame - the middle line on the top bar must be seen as bounding both the top and bottom edge of the front plane of the upper bar! According to Hochberg, the minimum principle should predict that this "contradiction" would yield organization of Figure $10 \mathrm{~A}$ as a flat line drawing, whereas it tends to be seen as a figure in depth. Hochberg (1982) attributed the depth result to the distance separating the left and right sides of Figure 10A, which allows each end to be organized in depth without forcing one to take in the contradiction. He contended that this interpretation is supported by the fact that Figure 10B tends to be organized bidimensionally, a finding that does not result merely from its dimensions, because Figure $10 \mathrm{C}$ elicits the perception of depth.

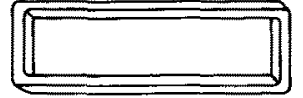

A

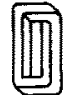

B

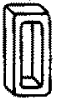

C
Figure 10. Two "impossible" figures (A and B); one "possible" figure (C). (Pattern A tends to elicit a stronger depth response than Pattern $B$, which cannot be due solely to Pattern B's small size, because Pattern C elicits a depth response. From "How Big is a Stimulus?" by $\mathbf{J}$. Hochberg. In Organization and Representation in Perception [p. 192] edited by J. Beck, 1982, Hillsdale, NJ: Erlbaum. Copyright 1982 by Erlbaum. Published with permission of Lawrence Erlbaum Associates.)

Hochberg's (1974, 1982) arguments based upon Figure 10 and other line drawings underscore the need to address the question of how great a segment of the display must be included for purposes of calculating simplicity (e.g., whether this should be set as a fixed extent within the visual field or determined on the basis of segmentation of the environment into objects). However, it is difficult to see how such figures confute explanations of form perception based on a minimum principle. Indeed, Simon (1967), in presenting the process model that we reviewed, explicitly considered the analysis of "impossible" figures. He suggested that the depth response to such figures arises because they are locally interpretable as consisting of tridimensional structures with rectangular sides. Once the "contradiction" is detected (Step C in his model), the depth response is maintained by restricting the "scanning" portion of the process to the noncontradictory portions of the stimulus. More recently, Perkins (1982) suggested that the depth response in "impossible" figures results from a "partial determination" of perceptual organization by the stimulus features. The depth response is elicited despite its "contradictoriness" because the perceptual system only takes a part of the stimulus into account at a given time. Thus, although Hochberg's arguments regarding "impossible" figures do not show that application of a minimum principle within perceptual theory would be "misguided" (1982, p. 195), they do emphasize the need to take into account the effective size of the stimulus in determining the relevant "geometric constraints" within which an economical configuration may be defined. 


\section{Choice of Simplicity Metric}

The idea that the perceptual system operates according to a minimum principle is open to a number of interpretations, depending on how one construes the notions of simplicity and regularity, and on what one thinks is minimized by the perceptual system. We have seen that our authors generally interpreted their minimum tendencies or minimum principles as applying to limited extents of the spatial layout, or to alterations in spatial configuration over a brief period of time. In focusing on this interpretation, we leave aside possible construals of the minimum principle as a broad principle of cognitive economy, that is, as a principle for achieving the simplest overall description of the environment (say, by achieving the simplest taxonomy of the objects in the environment).

Yet the idea of perceiving a minimum configuration may itself require reference to a description of the configuration and its alternatives. At least from the investigator's point of view, in order to characterize and to compare the simplicity of various configurations some metric of simplicity must be applied, and its application must involve at least a rudimentary description of the target objects. We have seen that a commonly adopted approach is to count and compare the number of primitive features that various configurations comprise (the fewer features, the simpler the configuration). This counting presupposes a taxonomy of features and, hence, a descriptive language. Because a given configuration may be described in more than one way, depending on what one chooses to call a feature, the theorist is faced with a problem of choosing the appropriate simplicity metric.

An example may help. Consider the four projections of a cube in Figure 1. Hochberg and Brooks (1960) devised 17 different measures of simplicity to be applied to such drawings. The relative simplicity of the bidimensional shapes to one another and the relation of the measured simplicity of these shapes to that of the tridimensional cube vary depending upon the measure adopted. In one of the measures, simplicity was determined by counting the number of continuous (but possibly transected) rectilinear segments in the figures; in another, by counting the number of unbroken line segments. (In each case, the lower the score, the simpler the figure.) The results for the four patterns are $12,12,11,9$, and $16,16,13,12$ for the respective measures. A tridimensional cube gets a score of 12 in either case. Notice that according to the first measure, the strong bidimensionality of Pattern $Z$ and slightly less strong bidimensionality of Pattern $Y$ is nicely set off from the other two patterns. According to the metric, the bidimensional organizations of these drawings clearly are simpler than those of the other two drawings, and perceivers are in agreement. Notice also that Patterns $W$ and $X$ have simplicity scores of 12 , which is equal to the score of the tridimensional organization. This leads one to expect an equivocation between the biand tridimensional organizations of these patterns, an expectation that is not borne out empirically. (The drawings tend to be organized as cubes.) A similar situation obtains with the second test. The simplicity measure suggests equivocation on Pattern $Z$, which in fact tends to be seen bidimensionally. Hochberg and Brooks did not make comparisons between scores for bi- and tridimensional organizations. They used the scores solely for comparisons of simplicity within sets of bidimensional shapes such as those in Figure 1. Our remarks are not meant to impugn this procedure, but rather to exemplify the fact that specific comparisons of simplicity are dependent upon the metric of simplicity that is chosen.

Although Hochberg and Brooks (1960) conveniently make our point for us by supplying alternative metrics, the point can be made with regard to any metric of simplicity. Thus, in the recent application of coding theory by Buffart et al. (1981) in assessing the simplicity of figural completions, it is clear that metrics other than those used by the authors could be proposed. This should be apparent from the fact that Buffart et al. applied their metric of simplicity in an entirely formal manner to the elemental symbols of the code itself. Thus, measured simplicity depends on what gets counted as a separate element of the code. For example, Buffart et al. (1981) did not include some elements of 
their code among the features to be counted to determine simplicity (e.g., operators that do not refer directly to elements of the target configuration). Their rationale was that these features "have only a notational meaning" (Buffart et al., 1981, p. 261). This decision is plausible only if one emphasizes simplicity of perceived form rather than economy of process. Further, they included no element in their code whatsoever for specifying that the completed figure is perceived as lying behind the occluding figure. Hence, the element of depth which is lacking in the mosaic interpretation received no weight in the completion interpretation. Nor is it obvious how much weight should be assigned to depth relative to other features, such as a line segment or an angle.

The studies that we have examined embody a variety of metrics. These include the following proposals for what gets minimized in perception: (a) the number of distinct features and relations among features, counted in one way or another (Buffart et al., 1981; Hochberg \& McAlister, 1953; Restle, 1979); (b) the variability among angles, line lengths, and line slopes (Attneave \& Frost, 1969); (c) the sum of distances traveled between successive frames among elements matched through apparent motion (Ullman, 1979); (d) the number of changes in the "common motion" component of a complex motion (Cutting \& Proffitt, 1982); and (e) the sum of elemental motions in the "relative motion" component of a complex motion (Cutting \& Proffitt, 1982). It was also proposed that perception tends toward qualitatively "good" figures, which were specified as figures with right angles or with symmetrical forms (Perkins, 1976). In addition, we have noted that Marr (1982), without connecting his work explicitly with the notion of a minimum principle, attributed to the visual system the assumptions that the environment contains smooth surfaces and relatively homogeneous optical textures. Consideration of this diverse group of simplicity metrics and qualitative specifications of Prägnanz reveals that the studies we have reviewed do not constitute a unified body of work investigating a common minimum principle or minimum tendency. Rather, these studies are only loosely related by their common use of such terms as "simplicity," "economy," "minimal," and "Prägnanz," together with the intuitive notions of simplicity or economy that lie behind the various proposed measures of such attributes.

Given the multiplicity of metrics, what should be the attitude of the investigator toward the choice of a metric of simplicity? Of course, the attitude may vary, depending on one's particular research strategies and goals. Hochberg and Brooks (1960), for example, were seeking to develop a purely psychophysical law that would predict subjects' responses. This led them to seek to establish empirically which of the 17 metrics of simplicity they examined best fit the behavior of subjects. The metric of simplicity they established would then act as a predictor, but need not reveal anything regarding the processes that lead to subjects' responses. It remains an open question whether the perceptual system registers as primitive those features that Hochberg and Brooks counted. With this approach, the only constraint on the choice of a metric of simplicity is the empirical adequacy of the psychophysical laws based on the metric.

Without denying the usefulness of a psychophysical approach, we wish to examine its limitations. The situation is parallel to the case of geometric constraints. Although the perceptual system may operate in such a way that it conforms to geometric optics, only facts about the processing mechanisms of the visual system can determine the extent to which perception actually matches geometric optics. Similarly, simplicity is not a direct datum available in proximal stimulation, nor is what is measured as "simplest" on a given simplicity metric necessarily that which an economizing perceptual system would deem simplest. A coding theory may predict the response of the visual system, but this can only be because the processing mechanisms of the visual system are what they are. The "simplest" construal of a given proximal pattern depends upon both the geometric constraints and the metric of simplicity implicit in the visual system's processing mechanisms, if there is one. Put another way, even though it is well established that arbitrarily many simplicity metrics can be constructed for assessing the simplicity of a range of phenomena, this fact does not imply that an econ- 
omizing visual system has access to a multiplicity of metrics. An answer to the question of whether the visual system embodies a unique simplicity metric depends upon facts about the system itself.

An arbitrarily chosen metric of simplicity might be brought by a program of empirical research into closer and closer match with the behavior of the visual system and ultimately might achieve predictive generality. A stronger program of research would be to regard the choice of a metric of simplicity as part of a hypothesis about the process mechanisms of the visual system. This would entail imputing a specific set of mechanisms for realizing geometric constraints and a specific simplicity metric to the visual system. This makes for a stronger hypothesis because it yields more routes of empirical testability. Any such hypothesis of course should be as accurate and as general a predictor as are the simplicity metrics of the psychophysical approach. In addition, particular structures are attributed to the visual system that can be expected to yield testable results along dimensions other than simplicity (e.g., reaction time or error curves). Moreover, if one actually imputes a coding system to the visual system, then it is natural to seek independent confirmation that the visual system registers the primitive features of the code.

\section{Competing Explanatory Foundations for the Minimum Tendency}

Recall that in the introduction we indicated that minimum tendencies have been established in the experimental literature independently of the idea that a minimum principle must provide the ultimate explanation for such tendencies. In fact, as an examination of contemporary studies reveals, a number of distinct explanatory foundations have been proposed for various minimum tendencies. We wish to examine the relation of the empirically observable minimum tendencies to these various explanatory frameworks. Our aim is not to find the one true explanatory framework. Certainly, the prime consideration is to determine which, if any, of the candidates are desirable, but this aim is complicated by the fact that the various frameworks are not universally incompatible with one another, nor do they all bear the same explanatory relation to minimum tendencies.

The types of explanations can be divided into two broad categories, corresponding to two distinct explanatory questions. The first group attempts to explain the presence of (or to "ground") specific minimum tendencies; these attempts differ according to how they answer the question of why the visual system has minimizing tendencies. The second group includes accounts of how perceptual economy is achieved; this is a question of process.

Grounding. Rival answers to the question of grounding divide into functional and nonfunctional accounts. Functional accounts stress the adaptive advantage of minimum tendencies, of which two have been proposed: (a) the adoption of a minimum principle yields veridical percepts because simplicity is a "good bet" (likelihood), and (b) economical representations make fewer demands on limited processing capacities (economy of process). On either of these proposals, the origin of a minimum tendency could in principle be attributed to either evolution or learning (or some combination of the two). As it happens, Perkins (1976), one adherent of the likelihood account, has assumed that likelihood is adopted as a good hypothesis on the basis of learning rather than through evolution. Conversely, economy of process as a fundamental strategy in perception is generally, but tacitly, regarded as arising through evolution (Mach, 1919, was explicit), although Vickers (1971, p. 27) has suggested that the strategy is learned.

The notion that a minimum tendency would be adaptive because of the high likelihood of simple configurations in the environment is difficult to assess. Earlier we noted that for a variety of well-known perceptual phenomena there is an intuitive match between the simplest and the most likely. Direct empirical evaluation of this seeming congruence would require the construction of precise measures of simplicity and likelihood and the development of suitable techniques for environmental sampling (Brunswick, 1956). The emphasis on likelihood may draw encouragement from the fact that a number of successful machine programs for modeling vision incorporate Prägnanz-like assumptions into their versions of the visual process. We have 
mentioned a number of Prägnanz assumptions in the work of Marr et al. In a similar vein, Barrow and Tenenbaum's (1978) model of the perception of surfaces, edges, and depth assumes the smoothness of surfaces. In these various cases, the investigators have built into the operation of their machine models assumptions to the effect that Prägnanz (smooth variation, homogeneous structures) is a good bet. The machine models were set to work on objects drawn from the same environment that confronts the human visual system (including conventional stimuli and ordinary objects). The fact that these programs yield veridical "percepts" argues for the functional validity of the Prägnanz assumptions in that environment. Hence, these assumptions may be regarded as functionally valid within the environment faced by the human visual system, although the successful operation of the machine programs does not establish that the human visual system actually embodies these assumptions.

An intriguing conception of the relation between likelihood and simplicity is suggested by the philosophical work of Goodman (1972, chap. 7) and Sober (1975). It has been shown that inductive sampling of the type that must underlie a judgment that the simpler is the more likely itself relies upon an implicit metric of simplicity. That is, extrapolation of a probability or likelihood judgment from a sample depends on a preference for fitting simple curves to the data from the sample. (Such a conception is at the core of Attneave's, 1954, discussion of redundancy and simplicity.) This might suggest that simplicity and likelihood are two faces of the same coin, and one need not treat the minimum principle and the likelihood principle as real alternatives. However, although the relation between inductive likelihood and simplicity is well established, it does not entail a blending of the concepts for present purposes. For although inductive sampling must indeed rely upon an implicit metric of simplicity (e.g., in curve fitting), the various metrics of simplicity that may be imputed to the visual system need not directly reflect the implicit simplicity metric (or metrics) behind the empirical practices of scientific investigation. It is empirically conceivable that environmental sampling as suggested by Brunswik
(1956) would show that the simpler (as defined on a given metric) is not the most likely. Here, the metric of simplicity that underlies the inductive sampling (curve fitting) can be quite independent of that which is used to measure the simplicity of environmental forms. The question of whether the visual system might tend toward simplicity on the basis of likelihood, or whether it does so on other grounds, retains its meaning.

Economy of process provides an alternative conception of the grounds for the minimum principle. There is intuitive appeal to the notion that cognitive systems are built so as to carry out cognitive processes with relative economy. This assumption was explicitly discussed by Mach $(1906,1919)$, who advocated a "biological-economical" orientation to the psychology of perception and cognition on evolutionary grounds. Mach argued that economy of thought would be of clear survival value to an organism faced with a bewildering array of sensory inputs, and he suggested that principles of economy operate from the lowest levels of sensory organization right up to the intellectual processes of the scientific investigator. The actual means for economizing are not given by the evolutionary approach as such; they depend upon the evolutionary history of the organism and must be investigated separately for different types of sensory systems. What the evolutionary approach does as a whole is provide a schema for answering the question of why the visual system economizes, thereby providing a conception of the functional role of minimum tendencies. The specific adaptive features of minimum tendencies also are not specified by an evolutionary approach as such, and they must be investigated for each type of sensory system in relation to its environment. Similar remarks apply to the programmatic suggestion that the minimum principle is an acquired strategy of cognitive economy (Vickers, 1971).

Finally, the chief exemplar of a nonfunctional grounding of the minimum tendency is that of the Gestalt psychologists, who derived the economy of perceptual configurations from the physico-chemical structure of processes in the brain. As conceived especially by Köhler (1920, 1947), these brain processes were regarded as occurring in gradient media 
or ionic "fields" in which the tendency toward minimal configurations is governed by the variational principles of classical mechanics (Planck, 1915, Lecs. 2, 7; Yourgrau \& Mandelstam, 1968). The appeal to spontaneous organizational forces in a cortical medium might seem implausible given what we know about the highly articulated architecture of the visual cortex. Köhler (1969, chap. 2) presented arguments to the contrary. $\mathrm{He}$ observed that even in highly articulated tissue, certain processes (e.g., steady-state physicochemical processes in the intercellular media) are not explained histologically. He contended that the evolution of articulated structure cannot explain facts about the physiology of organisms that result from the basic laws of physics and chemistry applied to the media surrounding these structures.

Köhler's contention cannot be dismissed out of hand. It accords with the notion, emphasized by evolutionary thinkers, that "mechanically necessary" aspects of an organism (such as the mass of a flying fish, which "functions" to bring it back to the water) are not to be treated as evolutionary adaptations (Williams, 1966, chap. 1). Moreover, appeal to "fields" or gradient media in animal tissue has long formed part of the theoretical landscape in developmental embryology (Köhler, 1927; Weiss, 1939, Part III, pp. 289-294), and it has been suggested that such fields exhibit simple, universal laws across a broad range of animal species, including hydra, sea urchins, fruit flies, newts, and chickens (Gierer, 1977; Kauffman, Shymko, \& Trabert, 1978; Wolpert, 1970). Schwartz (1977) extended this line of thinking to the development of receptotopic structures in the brains of such diverse species as the monkey and the goldfish. He proposed a set of "minimal developmental rules" that treat the developing neural structures as conforming to variational equations of the sort familiar within classical mechanics, and he commented that these rules "allow the final detailed structure of the receptotopic mapping to be determined by general physico-mathematical principles rather than via biological encoding of detailed positional information" (Schwartz, 1977, pp. 670-671). Consequently, it is a well-established pattern of thought within biology to seek to explain some phys- iological processes in terms of general physicochemical properties of intercellular media. Of course, because all known processes in organisms are in conformity with the laws of physics and chemistry, an evolutionary account may still be required to explain why certain processes following these laws are found in the brain and not others. Köhler's point that some of the processes underlying perception may reflect general physical conditions of animal tissue is not thereby negated, but its alleged independence from evolutionary considerations is weakened. The presence of specific kinds of ionic fields in developing or in mature animal tissue is not like having mass (mass being a property of all material things above the atomic level). Thus, Köhler's "grounding" is perhaps not nonfunctional after all, but his type of explanatory account retains its distinctiveness on other grounds, as we discuss in our consideration of process accounts.

Process. The two chief types of account of how perceptual economy is achieved are: (a) the so-called "soap bubble" accounts (Attneave, 1982), which explain perceptual economy in terms of physico-physiological processes, and (b) accounts that treat perception as the formation of internal descriptions (encoded propositionally) and regard perceptual economy as resulting from operations that yield economy of description. This division marks a distinction between two more general strategies in giving process accounts for perception: appeal to the brute properties of neurophysiological processes (as is often done to explain color vision and phenomena such as Mach bands), and appeal to the notion that perceptual processing occurs in an internal symbol system (or language of thought; Fodor, 1975), which serves as the vehicle for generating descriptions of the environment in accordance with encoded rules and strategies.

The Gestalt psychologists forthrightly posited physico-chemical mechanisms for achieving the minimal "solutions" to various perceptual "problems" (Koffka, 1919; Köhler, 1920, 1969; Wertheimer, 1912). According to Gestalt theory, the process underlying simplicity in perception is unmotivated and noncomputational. Any computation of simplicity is carried out by the scientist who wishes 
to predict the percept or assess the fit between the obtained and theoretically expected percept. Just as various physical systems achieve equilibrium at minimum states as a result of purely physical interactions, so too the nervous system achieves such solutions to perceptual problems by physico-chemical processes. This account has the advantage that it appeals to well-known processes for achieving minimal states or configurations.

The particular model of brain activity advocated by the Gestalt psychologists has long since been discarded, and a new account of the minimum process based on contemporary knowledge of the brain has not been formulated. Nevertheless, a number of contemporary investigators have concluded that an excessively atomizing approach to physiological psychology is bound to fail, and that the problems of neuropsychology require "confronting the theoretical and experimental perspectives demanded by the global, statistical, or Gestalt aspects of the nervous system" (John \& Schwartz, 1978, p. 25). Within neurophysiology, Schmitt, Dev, and Smith (1976) reviewed a large body of anatomical and electrophysiological data in support of the view that "graded electrotonic potentials, rather than regenerative spikes, may be the language of much of the central nervous system" (p. 116); they emphasized the role of "the extracellular electric field and ionic environment" (p. 117) in brain activity, while not denying that regenerative spikes retain their importance. From the side of mathematical biology, Cowan and Ermentrout (1978) developed mathematical models that treat the neural nets underlying perception "in terms of the properties of nonlinear fields or continua, which we introduce as a suitable representation of the activity seen in neural nets comprising large numbers of densely interconnected cells" (p. 69). The field concepts used by these authors must be sharply distinguished from extracellular fields, for they comprise interactions among numerous discrete neurons connected into nets. These various developments, taken together, suggest that the type of process account envisioned by Gestalt psychology cannot be dismissed.

Attneave (1982) recently undertook an interesting speculative exercise to reexamine the prospects of developing a conceptualiza- tion of perception in the spirit of the soapbubble metaphor favored by Gestalt theory. The soap bubble is an exemplar of systems "that progress to equilibrium states by way of events in interconnected and recursive causal sequences so numerous that their effects must be considered in the aggregate rather than individually" (Attneave, 1982, p. 12). Attneave's model is designed to explain monocular depth perception by positing internal regulatory tendencies which operate in a neuronal manifold representing external space. Perceptual organization is explained by appeal to roughly parallel organizational properties in the neural medium. In this case, phenomenal simplicity and regularity depend directly upon the simplicity and regularity of the structure of physiological processes. Economy of process accords with economical perceptual organization.

The chief alternative to the soap-bubble account is based on the notion that perception involves generating a description of the environment, and that economy of perception arises from economy of description. The notion of perception as description suggests the existence of a representational medium in which various alternative descriptions can be generated and evaluated for simplicity. As was discussed earlier, there are two basic versions of this process of generation and testing: selective and directive. The selective model suggests that the perceptual system examines a number of the permissible perceptual representations compatible with the optical constraints and selects from among these representations the alternative that passes a computational test for maximum economy. In contrast, the directive model suggests that the minimum principle guides the microgenesis of the perceptual representation to ensure the construction of the most economical perceptual representation.

Elaboration of a selective model requires consideration of a variety of subprocesses or component operations relating to the generation, assessment, and selection of alternatives. The decisions concerning these operations will shape the model. The following brief account is intended as illustration. In the initial stage, a number of the representations compatible with optical stimulation are generated simultaneously. Then the information 
load of each representation is computed concurrently according to a metric of simplicity that is imputed to the perceptual system. The computed values are scanned, and all representations but the one having the lowest informational load are cleared from working memory. This representation then serves as the representation of the distal configuration.

The initial stage of this or any other selective model is the most troublesome. How many candidate representations are to be generated, and how are they chosen? If the range of possibilities is continuous, clearly they cannot all be generated. The system might be set to calculate all possibilities separated by an arbitrarily chosen unit of simplicity, but even this suggests an overwhelming task of calculation. A similar problem is familiar to designers of artificial intelligence systems. As McArthur (1982) pointed out, a general problem faced by computer models of vision is the "combinatorial explosion" that arises when numerous possibilities are defined by a given visual input. Various strategies have been developed for narrowing down the alternatives. Minsky's (1975) frame theory is one such alternative. A selective model might be supplemented by the use of knowledge structures or "frames" that take into account the immediately prior perceptual situation to limit the generation of alternatives for simplicity testing. In this manner, perceptually untenable or unlikely representations are weeded out early. In any event, there are a number of empirical implications of this selective model which, if substantiated, would contribute to its plausibility. Given that the model specifies that the alternatives are subjected to evaluation, the percipient might be expected to have access to information about the rejected (less economical) representations. Second, if the observer is forced to make another choice, the perceptual representation ranked next in informational load should emerge.

We now turn to models of the directive variety. Several variants of the directive model may be formulated. One variant is suggested by Attneave's (1972) reference to a feedback or "hill-climbing" system, although the following account should not be attributed to Attneave. It is presumed that the perceptual system has evolved to favor the most eco- nomical representation compatible with the input. Upon reception of optical input, the perceptual system generates a first approximation perceptual representation from among the representations consistent with the input. The first approximation is followed immediately by transformation of the representation in an analog spatial representational medium. If the initial transformation generates a more economical representation according to a specified test, the process of transformation continues until it ceases to yield greater economy. It is assumed that once a gain in economy is realized the transformation proceeds in the same direction, so that there is no return to a less economical representation. If the initial transformation generates a less economical representation, the original representation is reinstituted. This serial process of testing ends when a specified number of successive transformations fails to yield a simpler representation.

Detailed development of a model along these lines will have to explicate several matters. As with the selective model, a decision rule is needed to specify the nature of the first approximation. This or a further rule must also be specified to effect the transformation process. Second, it will be necessary to arrive at a generalized metric of economy by which the gain or loss of economy is assessed. Because the assessment is executed by a human visual system, the metric should be one that may plausibly be attributed to the system. Third, the model must consider the comparison operation by which it is determined that a change in economy has occurred, and by which the process is terminated. As these steps are made explicit, the postulated process takes on an implausibly cumbersome visage.

An alternative to this hill-climbing approach is a directive model of the sort reviewed previously in connection with the work of Perkins (1976) and Simon (1967). The central idea is that the system seeks a fit among local features that concurs with Prägnanz (e.g., symmetry) and then propogates this interpretation throughout the scene, until an inconsistency is discovered or a coherent percept is obtained. As with models of a selective variety, a difficulty with this approach is "combinatorial explosion": a large 
number of initially promising but ultimately impossible interpretations may keep the system long at work. One way to avoid this problem is to build in especially strong constraints on the initial interpretation (Waltz, 1975). A mechanism for directly generating the simplest representation consistent with these constraints might then be provided by "filtering" or "constraint relaxation" techniques (McArthur, 1982). These operate in parallel to check the permissible combinations among local features according to the characteristic of the filter, which in this case seeks minimum configurations.

Significant increases in power are achieved by iteration of the operations (Barrow \& Tenenbaum, 1978; Rosenfeld, 1978). Indeed, by iterating the interactions among units receiving local inputs, global properties can be computed, just as a soap film achieves the global property of minimum surface area through numerous local interactions. Local interactions propagated across a network to compute a global property have been appropriately characterized as "pseudo-local" operators (Ikeuchi \& Horn, 1981, p. 181). Inasmuch as such mechanisms depend upon a large number of local interactions that converge on a minimal solution, they may be viewed as the computational analogue of the isoperimetric processes upon which Gestalt models are based (cf. Barrow \& Tenenbaum, 1978, p. 15). In fact, Grimson (1981), working in the tradition represented by Marr (1982), developed a model of stereopsis in surface perception that draws upon variational principles to compute various "minimum" values (of change in surface orientation) in the process of arriving at a representation of a distal surface.

Adherents of the notion of perception as description, whether working with a selective or a directive model, often implicitly assume that there is a direct relation between economy of description and economy of process (e.g., Buffart et al., 1981). The seeming plausibility of this assumption stems from the idea that simpler figures or event configurations have fewer distinct elements (e.g., lines or angles), and hence have briefer descriptions that require less processing capacity. Yet the assumption cannot be accepted without question, as we have seen. The question of whether the two types of economy coincide cannot be decided simply by examining the descriptions or codes that describe the perceived figure (the end-product of perceptual processing). To evaluate economy of process, one must examine the full account of the processes required to arrive at the simplest perceptual description. In the case of coding theory, this account might include detecting and encoding redundancy, generating alternative codes for a single stimulus, reducing them to their simplest form, and comparing them. This does not have the appearance of an economical process, even if it does succeed in detecting economical forms.

Our review of process accounts suggests that the basic approach of the Gestalt psychologists retains its attractiveness. The basic attractiveness of the position is that it provides a way of conceiving how economy can be achieved through the direct interaction of a large number of mutually independent events, such as might be conceived to occur in the visual cortex through lateral connections (Gilbert \& Wiesel, 1983; Rockland \& Lund, 1983). Yet although this approach has intuitive appeal, it does not have the specificity of the economy-of-description approach, in which explicit, serial procedures (however cumbersome) for generating representations and applying a simplicity metric to them can be worked out and perhaps even realized in the form of a computer program. However, investigators in biology have been developing mathematical models of brain activity that retain the local interactionism of the Gestalt approach, are plausibly considered to model actual brain processes, and bring the precision of mathematical modeling to this domain without invoking a computational metaphor. Such models have been applied to the activity of neural nets in general (Cowan \& Ermentrout, 1978), to the Gestalt rules of perceptual organization (Cowan, 1980, pp. 51-52), and to visual hallucinations (Ermentrout \& Cowan, 1979).

The related notion of massively parallel computational architectures provides an additional means for modeling processes that "compute" mathematically precise functions. Discussions of parallel computational architectures (Anderson, 1983; Anderson \& Hinton, 1981; Feldman, 1981; Hinton \& 
Sejnowski, 1983) stress the idea that "computations" may be conceived as direct interations among a large number of local units in the brain. This conception of computation may be viewed as a formalized version of two traditionally distinct traditions of thought: It is in the spirit of the qualitative Gestalt ideas about brain function as introduced by Köhler and his colleagues (Koffka, 1919, 1935; Köhler, 1920, 1947, 1969) and alluded to by Restle (1979), and it may be seen as a descendent of the connectionist approach represented by Hebb (1949). By envisioning massively parallel interactions, this approach can use conceptions of processing that approach the continuous variation of soap film.

There is great promise in massively parallel computational architectures for modeling cognitive functions (on vision see Ballard, Hinton, \& Sejnowski, 1983). Several varieties of parallel computational architectures are under investigation (Fahlman, Hinton, \& Sejnowski, 1983). Among the simpler of these is the "value-passing" architecture, in which computation is performed by numerous "units" or "nodes" through their mutual links. Such links can be excitatory or inhibitory and can vary in "gain." Input sets up a pattern of activity across a given (perhaps proportionally large) set of units. "Computation" occurs as these units mutually interact. The "output," or computed "representation," is constituted by the pattern of activation in the units once they have "settled down" to an equilibrium state. The subsequent use of this "representation" by other distinct processing systems would depend upon the interaction of this computational net with these other systems via connections originating in numerous local units.

As an example, consider a recent computational model of the process of deriving the shape of a surface from its projected image alone. The model was developed by Brown, Ballard, and Kimball (1982) and is in the spirit of Marr's (1982) approach as previously discussed (see also Horn, 1977). Analysis begins with reception of the shaded image of an object. Knowledge of the reflectance function of the surface is provided a priori. The system then computes a representation of shape from the image through massively parallel, cooperative processes that compute shape from numerous local inputs while interacting with a second computational system that computes the direction of the light source. This basic strategy was found to be robust over several variations in the details of the computational design.

Computational models that operate through the relaxation of parallel networks typically are minimizers. Ballard et al. (1983) described the computational task of deriving "shape from shading" as a "massive best-fit search." Insofar as this computational task is conceived as a best-fit problem, it is conceived as embodying a simplicity preference. Indeed, massively parallel computational architectures that compute through relaxation are commonly thought of as seeking a minimum energy state (Hinton \& Sejnowski, 1983). If the visual system is regarded as having such a computational architecture, then it must be regarded as an economizer. However, it need not be viewed as seeking "good forms" in the traditional sense of circles, squares, and rectangles. Rather, it seeks a good fit to numerous data points, where the best fit may be an irregular, asymmetrical form. The notion of "good form" in this case pertains to changes over small regions of the surface of an object (not to its global Gestalt properties) and may amount to no more than a continuous second derivative for the function describing local changes in surface orientation across an object. Thus, although the notion that visual processing draws upon a parallel computational architecture may lend support to the idea that visual processing incorporates a minimum principle, it does not necessarily support the notions of Prägnanz discussed by coding theorists or found in the qualitative approach of Perkins (1976, 1982). In this case, economy of process does not necessarily yield phenomenal simplicity.

We have treated the soap-bubble and the related massively parallel-architecture accounts as opposed to descriptionalist formulations. However, adherents of parallel architecture accounts sometimes characterize themselves as working within a descriptionalist framework (e.g., Marr, 1982), although others reject the notions of internal symbols (e.g., Feldman, 1981) and internal rules (e.g., Anderson \& Hinton, 1981). It may be argued that one strength of parallel architectures is 
the direct implementation of computational algorithms in the engineering of the system, without the need for internally represented rules or assumptions. If this point is accepted, the adoption of a soap-bubble or parallelarchitecture account need not preclude the idea that perception ultimately involves describing or categorizing objects in the environment. In fact, such a position leaves open the possibility of a hybrid conception of the relation between sensory perception and such cognitive operations as recognition. In this hybrid account any minimum tendencies in phenomenal experience would result from bottom-up processes working in relative independence of attention and semantic memory (as in Marr's, 1982, conception of early vision). These processes would yield representations of the spatial configurations of the environment that provide the basis for recognition, identification, and classification of objects.

\section{Conclusion}

When considered as a psychophysical law, the minimum tendency is well established for the perception of form, depth, and motion under certain conditions. Or perhaps one should say that diverse minimum tendencies have been established, because it is by no means clear that the various metrics of simplicity that have been applied to measure simplicity or Prägnanz in fact measure a single dimension or attribute. There may be as many minimum tendencies in perception as there are metrics of simplicity.

Granting, nonetheless, that there are tendencies toward perceptual economy, their theoretical implication remains uncertain. Our investigation leads us to believe that a global Minimum Principle, which acts as a cardinal principle of perception, will not be obtained. Nonetheless, the notion that a minimum principle (or principles) is operative in perception does raise interesting questions. Does it reflect a fundamental tendency of cognitive and perceptual systems to prefer simplicity? Is a preference for simplicity adaptive? Does it result from the fact that the simpler is the more likely? These questions remain open.

The idea that a tendency toward perceptual economy would be cognitively advantageous is appealing. Part of its appeal is derived from the notion that simplicity is adaptive because simpler representations take up fewer cognitive resources. This seems plausible for representations that are already in place. However, perception is a matter of generating representations as a consequence of stimulation. We have seen that if the representation generated is regarded as occurring within a symbolic medium as in coding theory, there currently is no basis for supposing that economy of description implies economy of process. In contrast, if phenomenal simplicity is explained within a soap-bubble account, simplicity of the structure of the process is correlated with perceptual economy. With such qualitative accounts, it is difficult to see the implications of the relationship between process and perception for overall cognitive economy, for it is unclear what to make of the relation between prägnant physiological structures and subsequent cognitive processing (e.g., recognition or memory). However, recent work that extends parallel computational models to higher domains (Anderson, 1983; Feldman \& Ballard, 1982) may provide a means for effecting this connection. Yet these types of models, which provide a precise characterization of economy of process, do not seem to favor economy of perceived form (global Gestalt properties), which weakens the proposed link between cognitive economy and phenomenal simplicity. One can only await further investigations of perceptual economy in which the metrics of simplicity and attendant process models are fully specified. Such investigations could provide a more definite specification of the ways in which perception tends toward the simple.

In the meantime, one is left with one's cognitive inclination toward perceptual economy. And that is just what wants explaining.

\section{References}

Anderson, J. A. (1983). Cognitive and psychological computation with neural models. Transactions on System, Man, and Cybernetics, 13, 799-815.

Anderson, J. A., \& Hinton, G. E. (1981). Models of information processing in the brain. In G. E. Hinton \& F. A. Anderson (Eds.), Parallel models of associative memory (pp. 9-48). Hillsdale, NJ: Erlbaum.

Attneave, F. (1954). Some informational aspects of visual perception. Psychological Review, 61, 183-193.

Attneave, F. (1972). Representation of physical space. In 
A. W. Melton \& E. J. Martin (Eds.), Coding processes in human memory (pp. 283-306). Washington, DC: Winston.

Attneave, F. (1982). Prg̈nanz and soap-bubble systems: A theoretical exploration. In J. Beck (Ed.), Organization and representation in perception (pp. 11-29). Hillsdale, NJ: Erlbaum.

Attneave, F., \& Frost, R. (1969). The determination of perceived tridimensional orientation by minimum criteria. Perception \& Psychophysics, 6, 391-396.

Ballard, D. H., Hinton, G. E., \& Sejnowski, T. J. (1983). Parallel visual computation. Nature, 306, 21-26.

Barrow, H. G., \& Tenenbaum, J. M. (1978). Recovering intrinsic scene characteristics from images. In A. Hanson \& E. Riseman (Eds.), Computer vision systems (pp. 3-26). New York: Academic Press.

Borjessen, E., \& von Hofsten, C. (1975). A vector model for perceived object rotation and translation in space. Psychological Research, 38, 209-230.

Braunstein, M. L. (1976). Depth perception through motion. New York: Academic Press.

Brown, C. M., Ballard, D. H., \& Kimball, O. A. (1982). Constraint interaction in shape-from-shading algorithms. In Proceedings of the DARPA Image Understanding Workshop (pp. 1-11). Springfield, VA: National Technical Information Service.

Brunswik, E. (1956). Perception and the representative design of psychological experiments. Berkeley, CA: University of California Press.

Buffart, H., Leeuwenberg, E., \& Restle, F. (1981). Coding theory of visual pattern completion. Journal of Experimental Psychology: Human Perception and Performance, 7, 241-274.

Buffart, H., Leeuwenberg, E., \& Restle, F. (1983). Analysis of ambiguity in visual pattern perception. Journal of Experimental Psychology: Human Perception and Performance, 9, 980-1000.

Cowan, J. D. (1980). Symmetry and symmetry-breaking in embryology and in neurobiology. In Ripon College studies in the liberal arts: Vol. 4. Concept formation and explanation of behavior (pp. 44-55). Ripon, WI: Ripon College Press.

Cowan, J. D., \& Ermentrout, G. B. (1978). Some aspects of the "Eigenbehavior" of neural nets. In S. A. Levin (Ed.), Studies in mathematical biology (Vol. 1, pp. 67117). Providence, RI: Mathematical Association of America.

Cutting, J., \& Proffitt, D. R. (1982). The minimum principle and the perception of absolute, common, and relative motions. Cognitive Psychology, 14, $211-$ 246.

Donnerstein, D., \& Wertheimer, M. (1957). Some determinants of phenomenal overlapping. American Journal of Psychology, 70, 21-37.

Duncker, K. (1929). Über induzierte Bewegung [On induced motion]. Psychologische Forschung, 12, 180259.

Ermentrout, G. B., \& Cowan, J. D. (1979). A mathematical theory of visual hallucination patterns. Biological Cybernetics, 34, 137-150.

Fahlman, S. E., Hinton, G. E., \& Sejnowski, T. J. (1983). Massively parallel architectures for AI: NETL, Thistle, and Boltzmann machines. In Proceedings of the Third National Conference on Artificial Intelligence (109113). Los Altos, CA: Kaufmann.
Feldman, J. A. (1981). A connectionist model of visual memory. In G. E. Hinton \& F. A. Anderson (Eds.), Parallel models of associative memory (pp. 49-81). Hillsdale, NJ: Erlbaum.

Feldman, J. A., \& Ballard, D. H. (1982). Connectionist models and their properties. Cognitive Science, 6, 205254.

Fodor, J. A. (1975). The language of thought. New York: Crowell.

Gibson, J. J. (1950). The perception of the visual world. Boston: Houghton Mifflin.

Gibson, J. J. (1966). The senses considered as perceptual systems. Boston: Houghton Mifflin.

Gibson, J. J. (1979). The ecological approach to visual perception. Boston: Houghton Mifflin.

Gierer, A. (1977). Biological features and physical concepts of pattern formation exemplified by hydra. Current Topics in Developmental Biology, 11, 17-59.

Gilbert, C. D., \& Wiesel, T. N. (1983). Clustered intrinsic connections in cat visual cortex. Journal of Neuroscience, 3, 1116-1133.

Goodman, N. (1972). Problems and projects. Indianapolis, IN: Bobbs-Merrill.

Gregory, R. L. (1974). Choosing a paradigm for perception. In E. C. Carterette \& M. P. Friedman (Eds.), Handbook of perception (Vol. 1, pp. 255-283). New York: Academic Press.

Grimson, W. E. L. (1981). From images to surfaces: $A$ computational study of the human early visual system. Cambridge, MA: MIT Press.

Hebb, D. O. (1949). Organization of behavior: $A$ neuropsychological theory. New York: Wiley.

Hinton, G. E., \& Anderson, F. A. (Eds.). (1981). Parallel models of associative memory. Hillsdale, $\mathrm{NJ}$ : Erlbaum.

Hinton, G. E., \& Sejnowski, T. J. (1983). Optimal perceptual inference. In Proceedings of the IEEE Computer Society Conference on Computer Vision and Pattern Recognition (pp. 448-453). Silver Springs, MD: IEEE Computer Society Press.

Hochberg, J. E. (1957). Effects of the Gestalt revolution: The Cornell symposium on perception. Psychological Review, 64, 73-84.

Hochberg, J. E. (1964). Perception (1st ed.). Englewood Cliffs, NJ: Prentice-Hall.

Hochberg, J. E. (1974). Organization and the Gestalt tradition. In E. C. Carterette \& M. P. Friedman (Eds.), Handbook of perception (Vol. 1, pp. 179-210). New York: Academic Press.

Hochberg, J. E. (1981). Levels of perceptual organization. In M. Kubovy \& J. R. Pomerantz (Eds.), Perceptual organization (pp. 255-278). Hillsdale, NJ: Erlbaum.

Hochberg, J. E. (1982). How big is a stimulus? In J. Beck (Ed.), Organization and representation in perception (pp. 191-217). Hillsdale, NJ: Erlbaum.

Hochberg, J. E., \& Brooks, V. (1960). The psychophysics of form: Reversible perspective drawings of spatial objects. American Journal of Psychology, 73, 337-354.

Hochberg, J. E., \& McAlister, E. (1953). A quantitative approach to figural "goodness." Journal of Experimental Psychology, 46, 361-364.

Horn, B. K. P. (1977). Understanding image intensities. Artificial Intelligence, 8, 201-231.

Ikeuchi, K., \& Horn, B. K. P. (1981). Numerical shape from shading and occluding boundaries. Artificial Intelligence, 17, 141-184. 
Johansson, G. (1950). Configurations in event perception. Stockholm, Sweden: Almqvist \& Wiksell.

Johansson, G. (1973). Visual perception of biological motion and a model for its analysis. Perception \& Psychophysics, 14, 201-211.

John, E. R., \& Schwartz, E. L. (1978). The neurophysiology of information processing and cognition. Annual Review of Psychology, 29, 1-29.

Julesz, B. (1971). Foundations of cyclopean perception. Chicago: University of Chicago Press.

Julesz, B. (1974). Cooperative phenomena in binocular depth perception. American Scientist, 62, 32-43.

Kauffman, S. A., Shymko, R. M., \& Trabert, K. (1978). Control of sequential compartment formation in Drosophila. Science, 199, 259-270.

Koffka, K. (1919). Zur theorie einfachster gesehner bewegungen. Ein physiologisch-mathematischer Versuch [The theory of the simplest perceived motions. A physiological-mathematical investigation]. Zeitschrift für Psychologie, 82, 257-292.

Koffka, K. (1930). Some problems of visual space perception. In C. Murchison (Ed.), Psychologies of 1930 (pp. 161-187). Worcester, MA: Clark University Press.

Koffka, K. (1935). Principles of Gestalt psychology. New York: Harcourt, Brace.

Köhler, W. (1920). Die physischen gestalten in ruhe und im stationären zustand [Physical gestalten at rest and in stationary processes]. Braunschweig, East Germany: Vieweg.

Köhler, W. (1927). Zum problem der regulation [On the problem of regulation]. Wilhelm Rowx' Archiv für Entwicklungsmechanik der Organismen. 112, 315322.

Köhler, W. (1947). Gestalt psychology. New York: Liveright.

Köhler, W. (1969). The task of gestalt psychology. Princeton, NJ: Princeton University Press.

Leeuwenberg, E. L. J. (1967). Structural information of visual patterns: An efficient coding system in perception. The Hague, Netherlands: Mouton.

Leeuwenberg, E. L. J. (1971). A perceptual coding language for visual and auditory patterns. American Journal of Psychology, 84, 307-349.

Leeuwenberg, E. L. J. (1978). Quantifications of certain visual pattern properties: Salience, transparency, similarity. In E. L. J. Leeuwenberg \& H. F. J. M. Buffart (Eds.), Formal theories of visual perception (pp. 277298). New York: Wiley.

Mach, E. (1906). Die analyse der empfindungen [The analysis of sensations] (5th ed.). Jena, East Germany: Fischer.

Mach, E. (1919). Die leitgedanken meiner naturwissenschaftlichen erkenntnisslehre und ihre aufnahme durch die zeitgenossen. Sinnliche elemente und naturwissenschaftliche begriffe. Zwei aufsatze [The key concepts of my natural-scientific theory of knowledge and their reception by contemporaries. Sensory elements and natural-scientific concepts. Two essays]. Leipzig, East Germany: Barth.

Mach, E. (1960). The science of mechanics (6th ed., T. J. McCormack, Trans.). La Salle, IL: Open Court. (Original work Die mechanik in ihrer entwicklung, 1883, Leipzig, East Germany: Brockhaus).

Marr, D. (1982). Vision. San Francisco: Freeman.

Marr, D., \& Hildreth, E. (1980). Theory of edge detection.
Proceedings of the Royal Society of London, B 209, 199-218.

Marr, D., \& Nishihara, H. K. (1978). Representation and recognition of the spatial organization of three-dimensional shapes. Proceedings of the Royal Society of London, B 200, 269-294.

Marr, D., \& Poggio, T. (1976). Cooperative computation of stereo disparity. Science, 194, 283-287.

Marr, D., \& Poggio, T. (1979). A computational theory of human stereo vision. Proceedings of the Royal Society of London, B 204, 301-328.

McArthur, D. J. (1982). Computer vision and perceptual psychology. Psychological Bulletin, 92, 283-309.

Minsky, M. (1975). A framework for representing knowledge. In P. H. Winston (Ed.), The psychology of computer vision (pp. 211-277). New York: McGrawHill.

Perkins, D. N. (1972). Visual discrimination between rectangular and nonrectangular parallelopipeds. Perception \& Psychophysics, 12, 396-400.

Perkins, D. N. (1976). How good a bet is a good form? Perception, 5, 393-406.

Perkins, D. N. (1982). The perceiver as organizer and geometer. In J. Beck (Ed.), Organization and representation in perception (pp. 73-93). Hillsdale, NJ: Erlbaum.

Perkins, D. N., \& Cooper, R. (1980). How the eye makes up what the light leaves out. In M. Hagen (Ed.), The perception of pictures: Vol. II. Durer's devices: Beyond the projective model of pictures (pp. 95-130). New York: Academic Press.

Planck, M. (1915). Eight lectures on theoretical physics (A. P. Willis, Trans.). New York: Columbia University Press.

Restle, F. (1979). Coding theory of the perception of motion configurations. Psychological Review, 86, 124.

Rock, I. (1975). Introduction to perception. New York: Macmillan.

Rock, I. (1977). In defense of unconscious inference. In W. Epstein (Ed.), Stability and constancy in visual perception (pp. 321-373). New York: Wiley.

Rock, I. (1983). The logic of perception. Cambridge, MA: MIT-Bradford.

Rock, I., \& Smith, D. (1981). Alternative solutions to kinetic stimulus transformations. Journal of Experimental Psychology: Human Perception and Performance, 7, 19-29.

Rockland, K. S., \& Lund, J. S. (1983). Intrinsic laminar lattice connections in primate visual cortex. Journal of Comparative Neurology, 216, 303-318.

Rosenfeld, A. (1978). Iterative methods in image analysis. Pattern Recognition, 10, 181-187.

Schmitt, F. O., Dev, P., \& Smith, B. H. (1976). Electrotonic processing of information by brain cells. Science, 193 , 114-120.

Schwartz, E. L. (1977). The development of specific visual connections in the monkey and the goldfish: Outline of a geometric theory of receptotopic structure. Journal of Theoretical Biology, 69, 655-683.

Simon, H. (1967). An information-processing explanation of some perceptual phenomena. British Journal of Psychology, 58, 1-12.

Sober, E. (1975). Simplicity. London: Oxford University Press. 
Uhr, L. (1982). Computer perception and scene analysis. In C. Y. Suen \& R. DeMori (Eds.), Computer analysis and perception (Vol. 1, pp. 1-16). Boca Raton, FL: CRC.

Ullman, S. (1979). The interpretation of visual motion. Cambridge, MA: MIT.

Vickers, D. (1971). Perceptual economy and the impression of visual depth. Perception \& Psychophysics, 10, 23-28.

Wallach, H. (1965). Visual perception of motion. In G. Kepes (Ed.), The nature and the art of motion (pp. 52-59). New York: Braziler.

Waltz, D. (1975). Understanding line drawings of scenes with shadows. In P. H. Winston (Ed.), The psychology of computer vision (pp. 19-92). New York: McGrawHill.

Weiss, P. (1939). Principles of development. New York: Holt.
Wertheimer, M. (1912). Experimentelle Studien über das Sehen von Bewegung [Experimental studies of the perception of motion]. Zeitschrift für Psychologie, 12, 161-265.

Williams, G. C. (1966). Adaptation and natural selection. Princeton, NJ: Princeton University Press.

Wolpert, L. (1970). Positional information and pattern formation. In C. H. Waddington (Ed.), Towards a theoretical biology (Vol. 3, pp. 198-230). Chicago: Aldine.

Yourgrau, W., \& Mandelstam, S. (1968). Variational principles in dynamics and quantum theory (3rd ed.). Philadelphia: Saunders.

Received November 7, 1983 Revision received June 6, 1984 